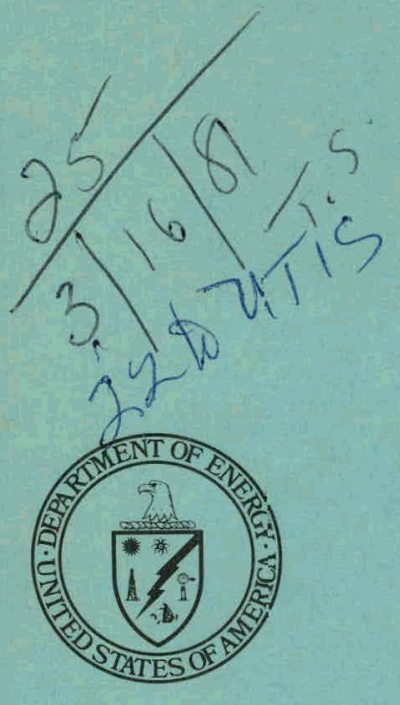

\title{
International
}

Energy Indicators

\section{U.S. Department} of Energy

International Affairs

Office of Market Analysis

February 1981

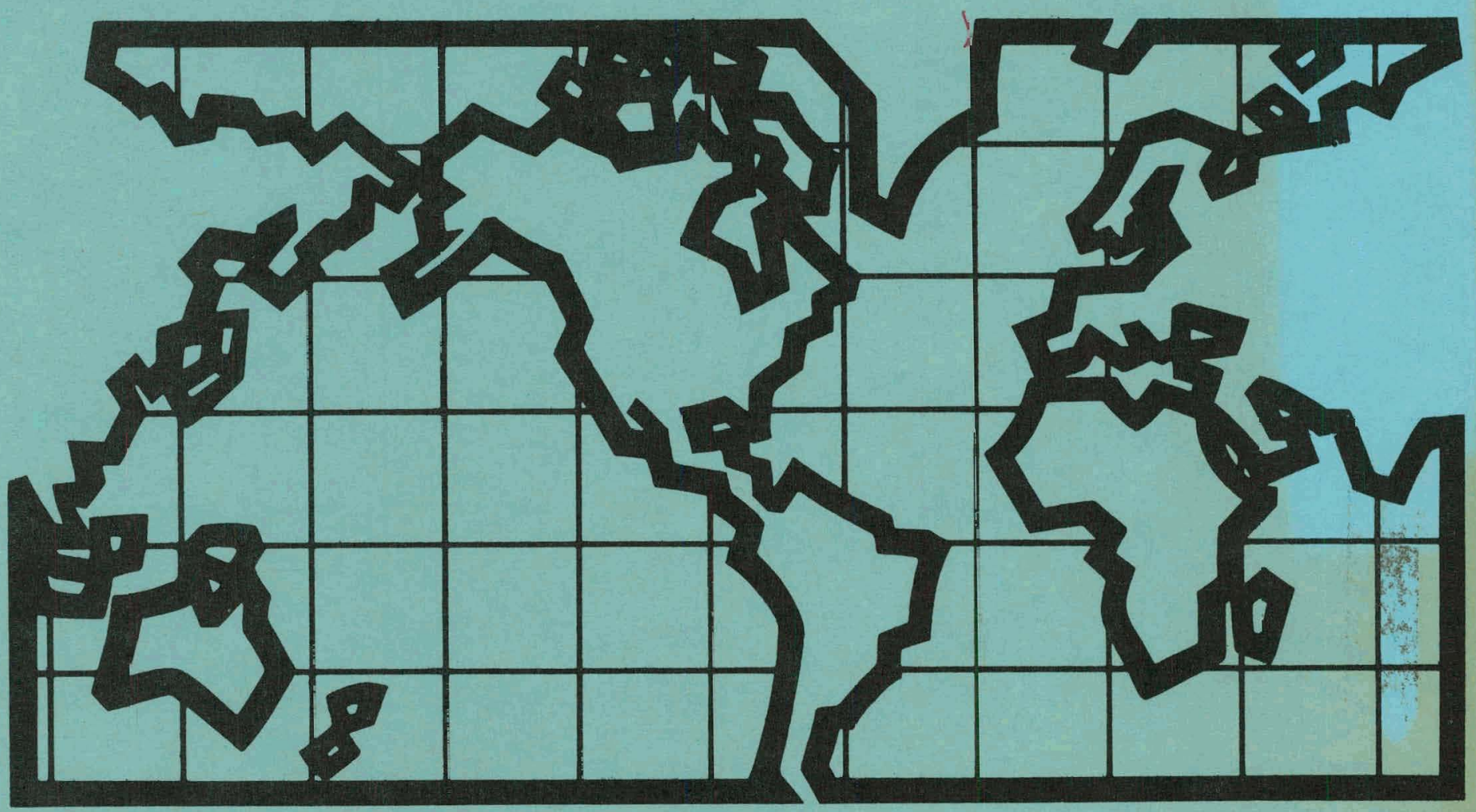




\section{DISCLAIMER}

This report was prepared as an account of work sponsored by an agency of the United States Government. Neither the United States Government nor any agency Thereof, nor any of their employees, makes any warranty, express or implied, or assumes any legal liability or responsibility for the accuracy, completeness, or usefulness of any information, apparatus, product, or process disclosed, or represents that its use would not infringe privately owned rights. Reference herein to any specific commercial product, process, or service by trade name, trademark, manufacturer, or otherwise does not necessarily constitute or imply its endorsement, recommendation, or favoring by the United States Government or any agency thereof. The views and opinions of authors expressed herein do not necessarily state or reflect those of the United States Government or any agency thereof. 


\section{DISCLAIMER}

Portions of this document may be illegible in electronic image products. Images are produced from the best available original document. 


\section{International Energy Indicators}

February 1981

U.S. Department

of Energy

International Affairs

Office of Market Analysis

Dr. Elizabeth K. Bauer, Editor, 202-252-5162, 7A-029 Forrestal Building

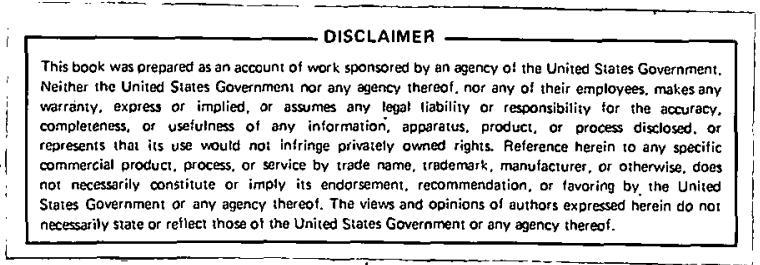




\section{Table of Contents and Highlights}

\section{INTERNATIONAL}

World Crude Oil Production, 1975 -

World crude oil production rose steadily from some $53 \mathrm{MMB} / \mathrm{D}$ in 1975 to $62.6 \mathrm{MMB} / \mathrm{D}$ in 1979 , with the OPEC countries accounting for the bulk of the gain in most years. In the first 11 months of 1980 , total world production dropped to $59.5 \mathrm{MMB} / \mathrm{D}$, and the level of OPEC production was slightly below its 1975 figure.

Iran: Crude Oll Capacity, Production and Shut-in, 1974 -

Estimated production rose to $1.5 \mathrm{MMB} / \mathrm{D}$ in December according to DOE/IA, though analysts are keeping capacity at about 3.0 MMB/D. Domestic consumption has been cut back to $.5 \mathrm{MMB} / \mathrm{D}$, and exports were around $1 \mathrm{MMB} / \mathrm{D}$.

Saudi Arabia: Crude Oil Capacity, Production and Shut-in, 1974 -

Saudi Arabia's production remained at about 10.3 MMB/D in December, with $.275 \mathrm{MMB} / \mathrm{D}$ coming from the Neutral Zone. The shut in was still close to zero.

OPEC (Ex-Iran and Saudi Arabia): Capacity, Production and Shut-in, 1974 -

The rest of OPEC was estimated to be producing $12.9 \mathrm{MMB} / \mathrm{D}$ in November with about $6.4 \mathrm{MMB} / \mathrm{D}$ shut-in.

Oil Stocks: Free World, U.S., Japan and Europe (Landed), 1973 -

Revised estimates for U.S. stocks stood at 1.38 billion bbl and Japanese at 469 million bbl for $4 Q 80$. DOE/IA estimates that world stocks for 4080 were 4.45 billion bbl. Western Europe data show stocks at 1.45 billion bbat in 4Q80. Note: 1980 figures expanded beyond "Big Five."

Petroleum Consumption by Industrial Countries, 1973 -

Figures for August 1980 show the industrial countries with a much lower seasonal rate of consumption than 1979down some $5.6 \mathrm{MMB} / \mathrm{D}$ of which the U.S. accounted for $1.9 \mathrm{MMB} / \mathrm{D}$, or one third of the total. The adjusted 12-month average for the IEA countries plus France continues the downward movement begun in November 1979

\section{USSR Crude Oil Production, 1974 -}

The graphic display shows the rate of change of USSR oil production, including natural gas liquids. For January, the rate dropped to 1.4. Annual export figures include shipments to both Bloc and Western countries.

Free World and U.S. Nuclear Generation Capacity, 1973 -

Free World capacity rose by $685 \mathrm{MW}$ in November when Finland added a 465-MW generator and India added a 220-MW unit. In December, France brought on two new generators totalling $1914 \mathrm{MW}$, and Sweden added a $940-M W$ unit for a grand total of 2854 new MW. The U.S. remained practically stationary. 
U.S. Gross Imports of Crude Oll and Products, 1973

in January, gross U.S. imports stood at $70 \mathrm{MMB} / \mathrm{D}$. The 12-month moving average, based on adjusted figures, was down to $6.5 \mathrm{MMB} / \mathrm{D}$.

\section{Landed Cost of Saudi Crude in Current and 1974 Dollars}

According to data derived from information provided to DOE, preliminary figures show that the landed cost of Saudi Light crude stood at $\$ 31.22$ in October. In 1974 dollars, the October price was $\$ 18.17$ representing a real increase between April 1974 and October 1980 of $\$ 6.27$.

\section{U.S. Trade in Bituminous Coal, 1973 -}

In December, exports fell to 8.1 million short tons of bituminous coal and imports were 70,000 short tons. The 12-month moving average for exports was 7.5 million short tons in December, representing a steady rise since 3078.

\section{Summary of U.S. Merchandise Trade, 1976 -}

The deficit rose in December to almost $\$ 3$ billion. Manufactured goods showed a surplus of almost $\$ 1.5$ billion and agriculture's surplus was $\$ 2.3$ billion. The deficit in energy rose to $\$ 6.78$ billion in December, up from $\$ 5.4$ billion in November.

\section{Energy/GNP Ratio}

Third quarter 1980 preliminary figures follow the normal seasonal pattern. The adjusted moving average was 53.22. 
World Crude Oil Production, 1975-

(In Thousands of Barrels a Day)

World

Non-Communist Countries

OECD

United States

Canada

Norway

United Kingdom

Other

Non-OPEC LDCS

Egypt

Mexico
Other $^{2}$

OPEC

Algeria

Ecuador

Gabon

Indonesia

Iran

Iraq

Kuwait

Libya

Neutral Zone

Nigeria

Qatar

Saudi Arabia

United Arab Emirates

Venezuela

Communist Countries

USSR

China

\section{$\begin{array}{lllll}1975 & 1976 & 1977 & 1978 & 1979\end{array}$}

$52,995 \quad 57,360 \quad 59,680 \quad 60,145 \quad 62,560 \quad 61$,

$\begin{array}{llllll}41,345 & 44,970 & 46,620 & 46,380 & 48,530 & 47\end{array}$

$8,375 \quad 8,130$$$
\begin{array}{r}
190 \\
20 \\
750
\end{array}
$$

$3,450 \quad 3,630$

230

2,500

2,500

27,140

$$
960
$$$$
160
$$$$
1,310
$$

$\begin{array}{ll}1,310 & 1,505 \\ 5,350 & 5,885\end{array}$

2,260

$1,840 \quad 1,910$

$1,480 \quad 1,935$

495
1,785

$\begin{array}{rr}1,785 & 2,065 \\ 440 & 495\end{array}$

$\begin{array}{ll}6,825 & 8,345 \\ 1,665 & 1,935\end{array}$

$2,345 \quad 2,295$

$11,650 \quad 12,390$

$1,540 \quad 1,735 \quad 1,87$

$480, \quad 485$

$\begin{array}{llll}8,245 & 8,705 & 8,550 & 8\end{array}$

$280 \quad 350$

$745 \quad 740$

185200

225
205

$\begin{array}{rrr}1,685 & 1,635 & 1,595\end{array}$
$1980^{1}$

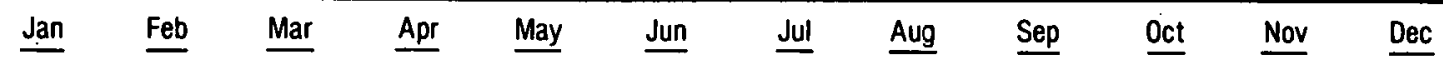

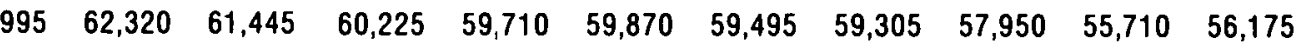

$7,900 \quad 48,2$

47,270

$46,060 \quad 45,475 \quad 45,705$

$\begin{array}{lllll}45,160 & 44,950 & 43,615 & 41,425 & 41,850\end{array}$

$3,030 \quad 13,110$

$3,110 \quad 12,870$

$12,895 \quad 12,805$

$12,625 \quad 12,650$

$12,595 \quad 12,660 \quad 12,540$

$\begin{array}{llll}1,495 & 1,480 & 1,485 & 1,695\end{array}$

8,690

8,555

$8,420 \quad 8,540 \quad 8,570 \quad 8,500$

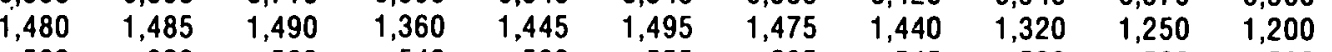

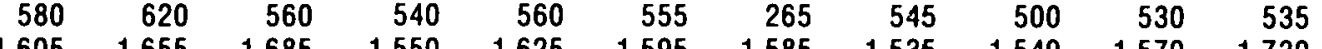

$\begin{array}{llllllllllll}1,570 & 1,605 & 1,655 & 1,685 & 1,550 & 1,625 & 1,595 & 1,585 & 1,535 & 1,540 & 1,570 & 1,730\end{array}$

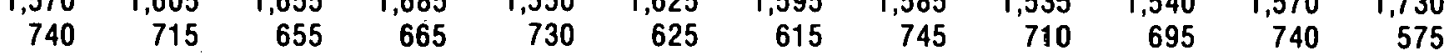

5,325

5,385

5,405

5,405

$5,500 \cdot 5,465$

5,560

$5,685 \quad 5,460$

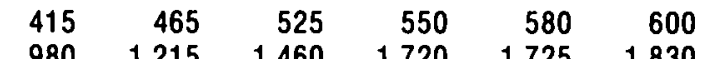

$600 \quad 600 \quad 600$

$600 \quad 575$

$\begin{array}{lll}575 & 605 & 625\end{array}$

$\begin{array}{rrrrrrrr}1,885 & 1,910 & 1,905 & 2,015 & 2,000 & 2,125 & 2,180 & 1,900\end{array}$

$2,900 \quad 2,895 \quad 2,900$

$2,885 \quad 2,890$

2,860

2,900

2,935

$29,91,0 \quad 28,835$

27,805

27,495

27,035

26,835

900
180
180

23,85

$\begin{array}{llll}1,095 & 1,160 & 1,175 & .1,200\end{array}$

180

1,200

230
180

190

1,000
250

1,000

1,000
230

900
220

900
240

$1,000 \quad 1,000$

1,565

1,550
2,500

1,575

1,550
1,800

180
1550

180
1,575

180
1565

180
1,565

180
1565

180

225

$\begin{array}{llll}2,350 & 2,560 & 3,435 & 3,500\end{array}$

$\begin{array}{ll}2,500 & 2,000 \\ 3,500 & 3,500\end{array}$

$1,800 \quad 1,300$

$\begin{array}{ll}1,800 & 1,300 \\ 1,500 & 3,500\end{array}$

1,500
3,500

1,500

1,300

1,565
1,100

2,900

$\begin{array}{llllll}1,785 & 1,895 & 2,215 & 1,850 & 2,035 & 1,770 \\ 2,065 & 1,980 & 2,065 & 2,100 & 2,100 & 2,000\end{array}$

$\begin{array}{ll}1,285 & 1,235 \\ 1,750 & 1,700\end{array}$

1,320

$-1,115$

1,145

$2,100 \quad 2,000$

540

1,700
555
2,140

1,700

1,680

1,690

1,050
1,680

$\begin{array}{rr}150 & 300 \\ 1,100 & 1,200\end{array}$

$\begin{array}{rrrr}390 & 475 & 565 & 585\end{array}$

$\begin{array}{rrrr}2,085 & 1,895 & 2,305 & 2,150 \\ 445 & 485 & 505 & 500\end{array}$

$2,150 \quad 2,155$

2,190

2,140
475

2,195

2,100

520

480

$1,665 \quad 1,680$

$\begin{array}{rrrrrr}9,015 & 8,065 & 9,250 & 9,500 & 9,500 & 9,500\end{array}$

9,500

$9,500 \quad 9,500$

460

470

1,600

$510 \quad 530$

$\begin{array}{llllll}2,000 & 1,830 & 1,830 & 1,740 & 1,740 & 1,695\end{array}$

1,710

$9,500 \quad 9,500$

$440 \quad 475$

$\begin{array}{lllll}2,165 & 2,355 & 2,280 & 2,205 & 1,990\end{array}$

$2,050 \quad 2,045$

2,060

2,170

2,210

$1,675 \quad 1,675 \quad 1,000$

$\begin{array}{lll}1,675 & 1,675 & 1,695\end{array}$

$\begin{array}{llllllll}14,165 & 14,235 & 14,165 & 14,335 & 14,355 & 14,335 & 14,285 & 14,325\end{array}$

11,640

11,630

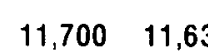

11,800

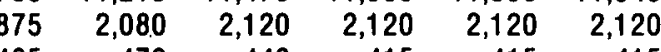

$2,120 \quad 2,120$

$2,120 \quad 2,120$

$2140 \quad 2,120 \quad 11,800 \quad 11,820$

41
4

${ }^{3}$ Albania, Butgaria, Cuba, Czechoslovakia, East Germany, Hungary, Poland, Romania, and Yugoslavia.

SOURCES: DOEJEIA, OPEC, CIA OHfice of Economic Research, Oil and Gas Joumal. 


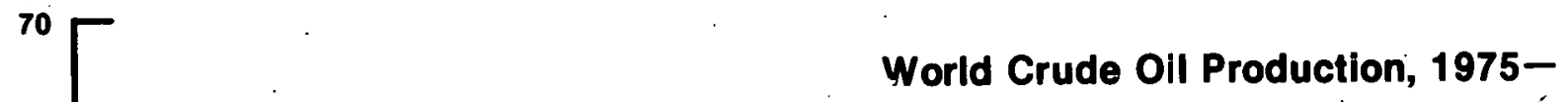

(In Millions of Barrels a Day)

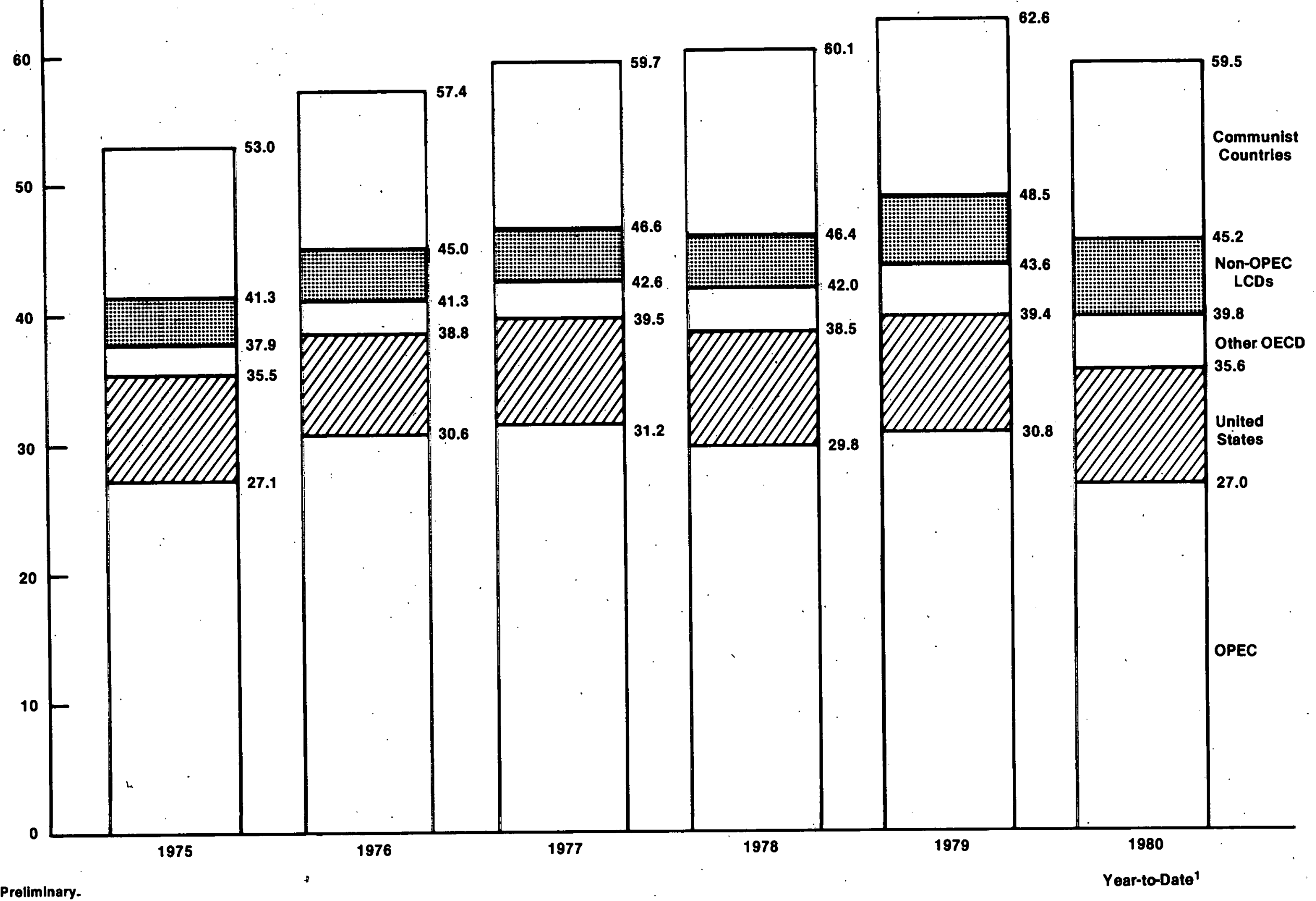


Iran: Crude Oil Capacity, Production and Shut-In, 1974 (In Thousands of Barrels per Day)

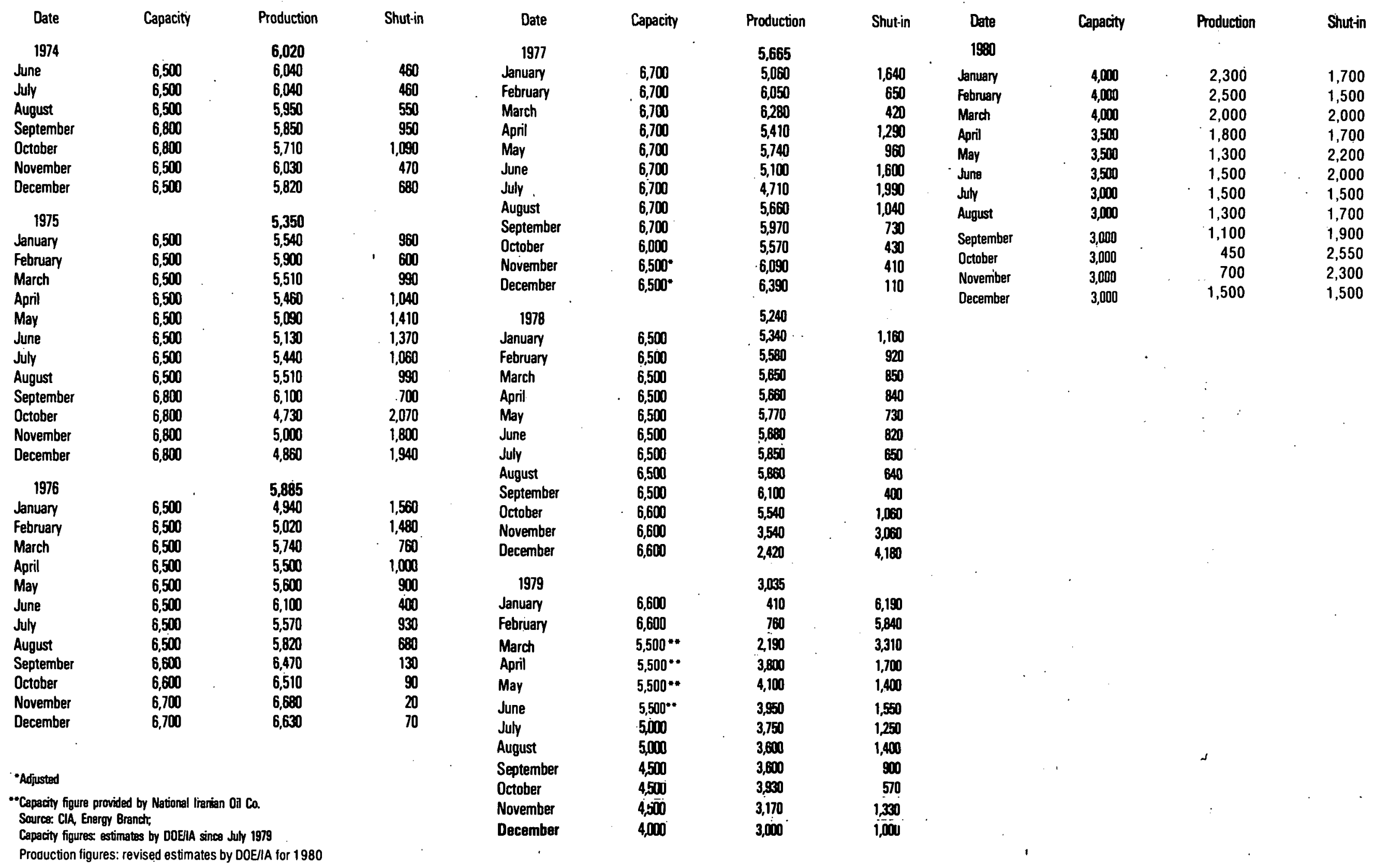


Iran-Capacity, Production and Shut-In, 1974 -

(In Millions of Barrels a Day)

MMB/D

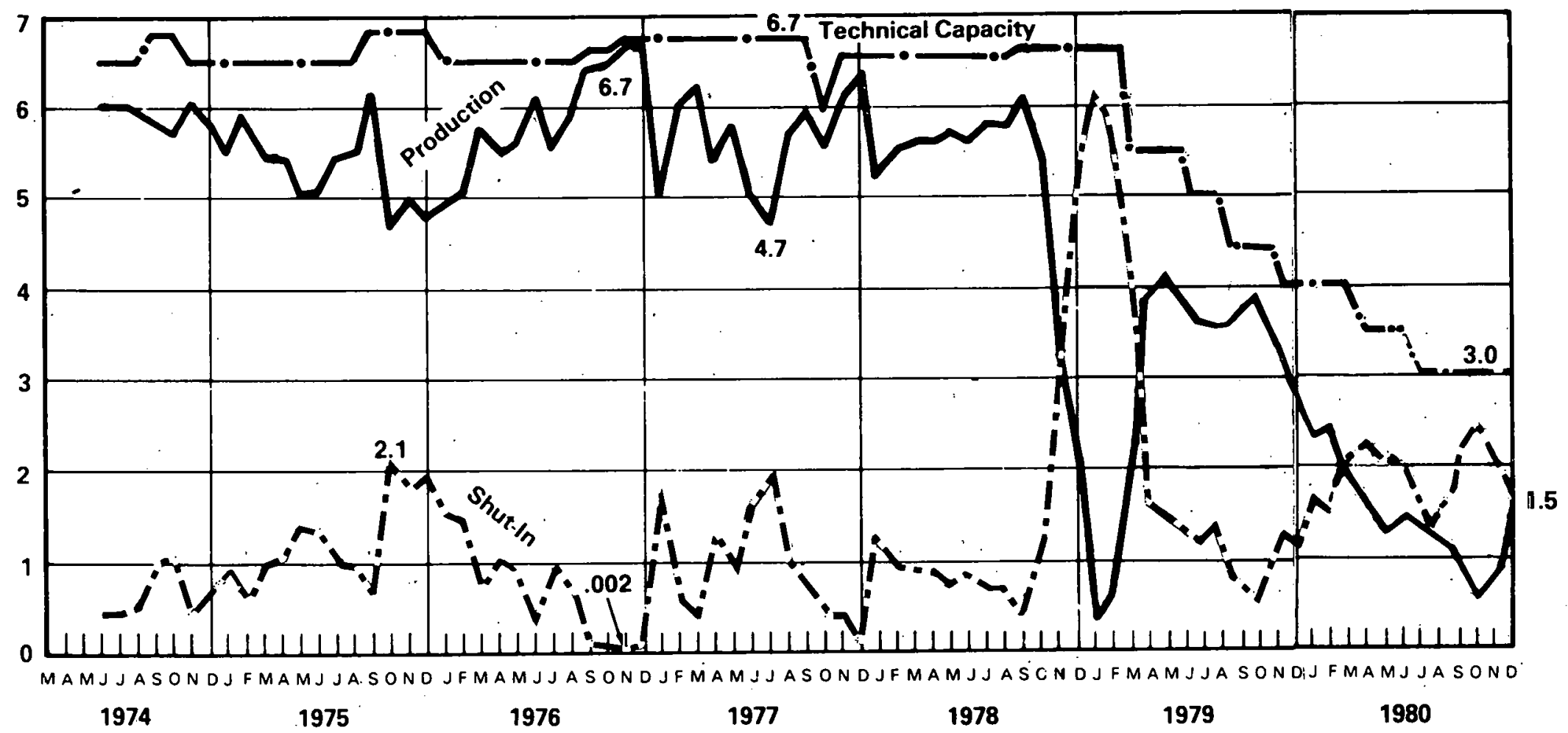


Saudi Arabia: Crude Oil Capacity, Production and Shut-In, 1974 -

(In Thousands of Barrels per Day)

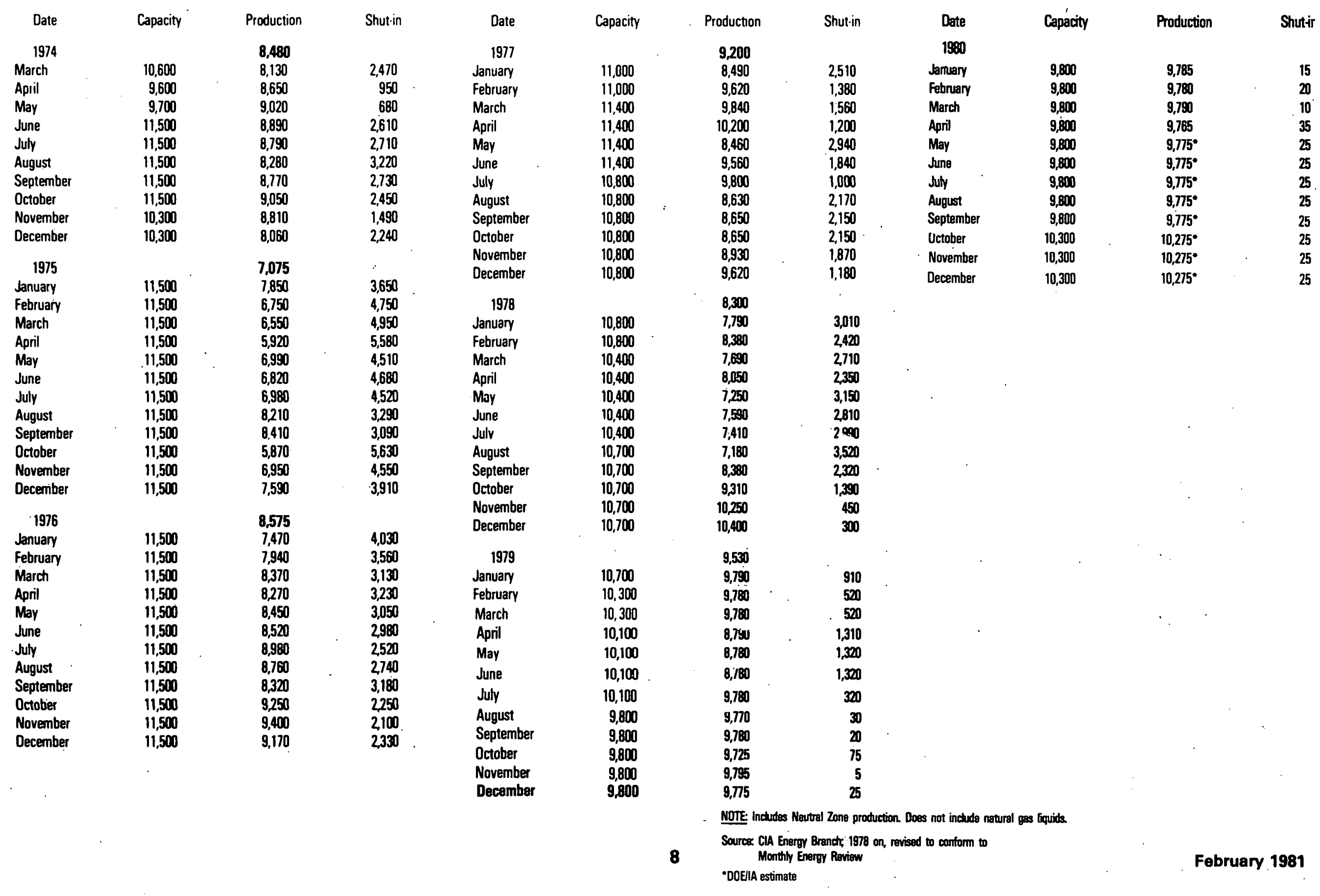


Saudi Arabia-Capacity, Production and Shut-In, 1974 -

(In Millions of Barrels a Day)

MMB/D

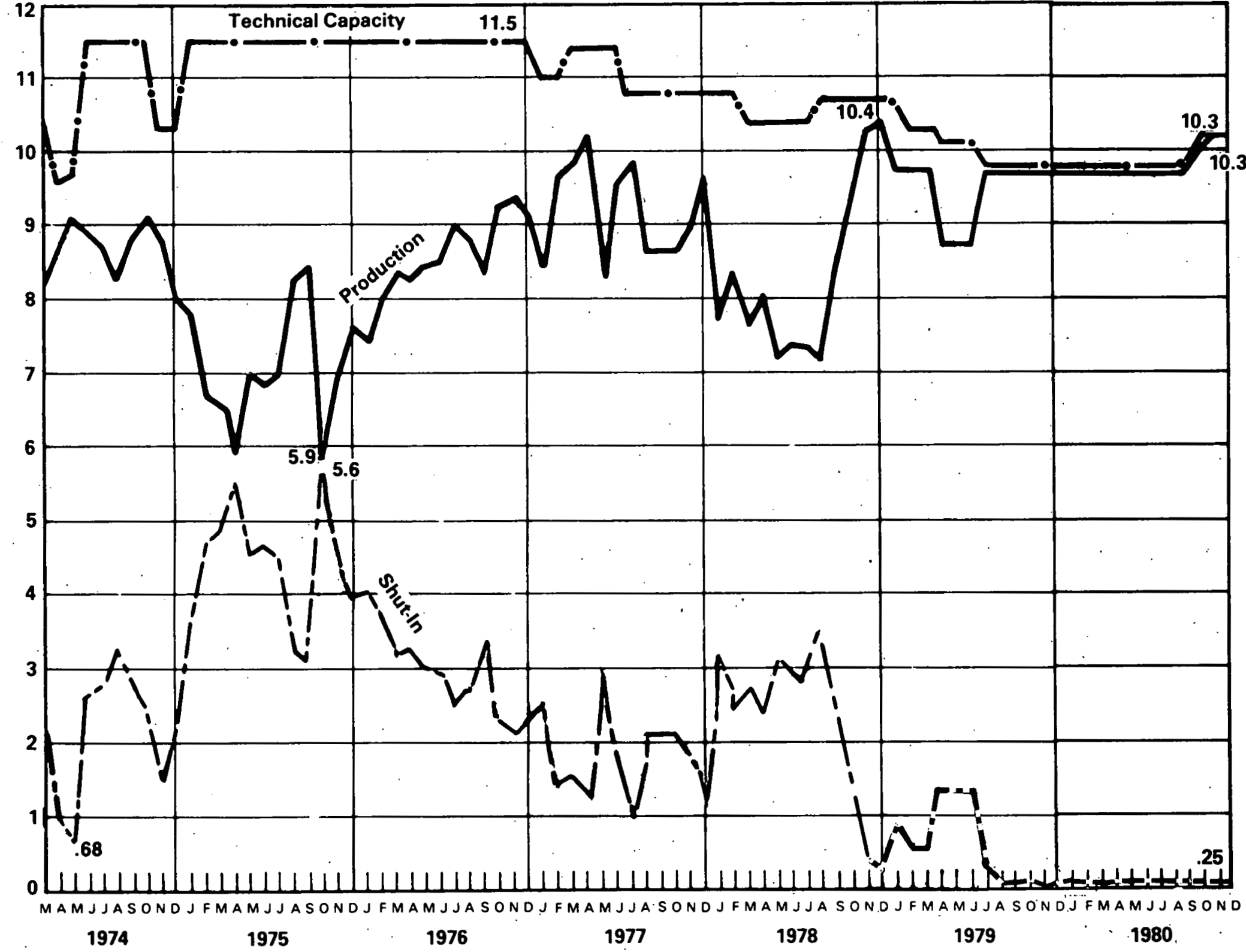


OPEC (Ex-Iran and Saudi Arabia): Capacity, Production and Shut-In, 1974 (In Thousands of Barrels a Day)

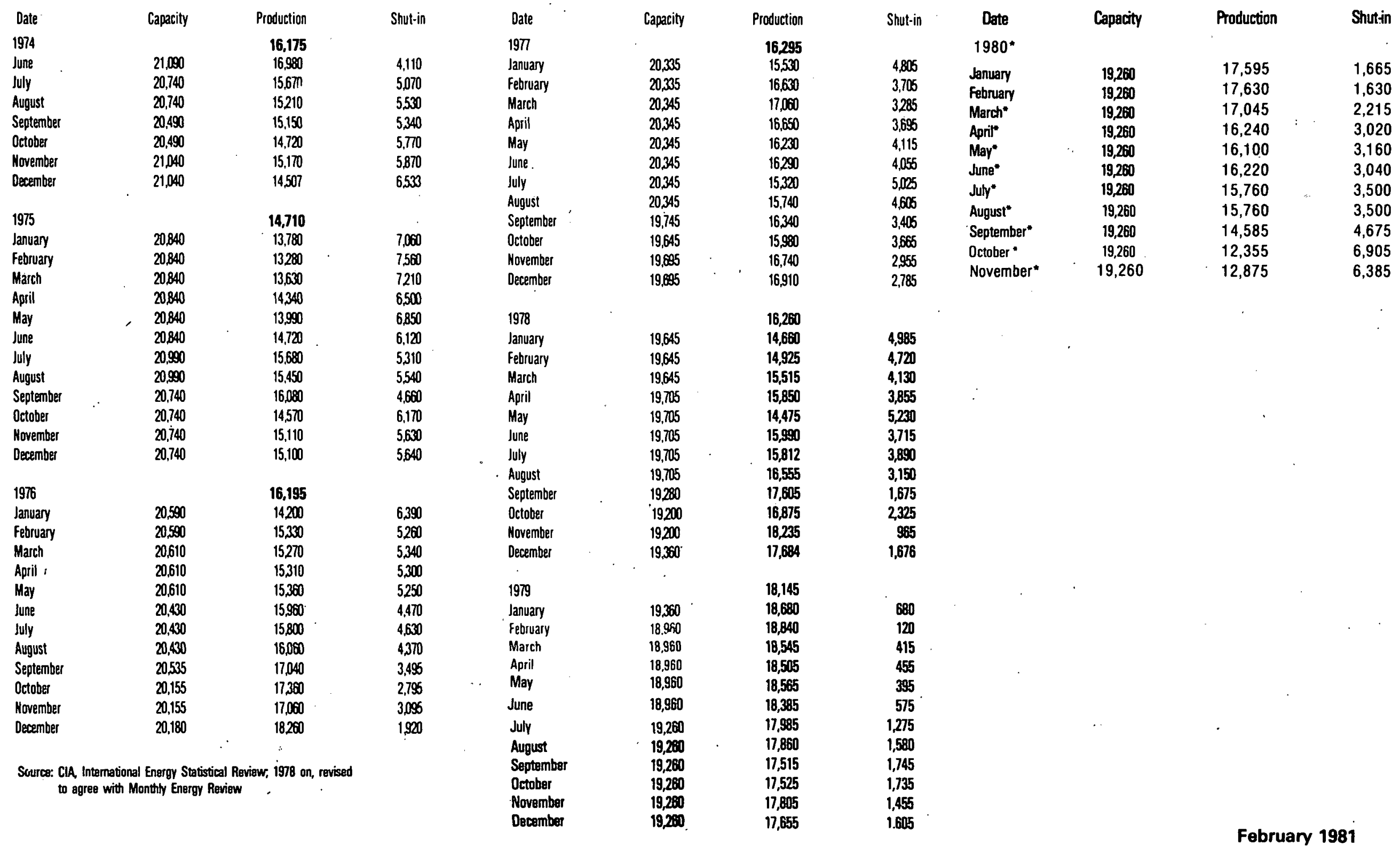


OPEC (Ex-Iran and Saudi Arabia) Capacity, Production and Shut-In, 1974-

(In Millions of Barrels per Day)

\section{MMBID}

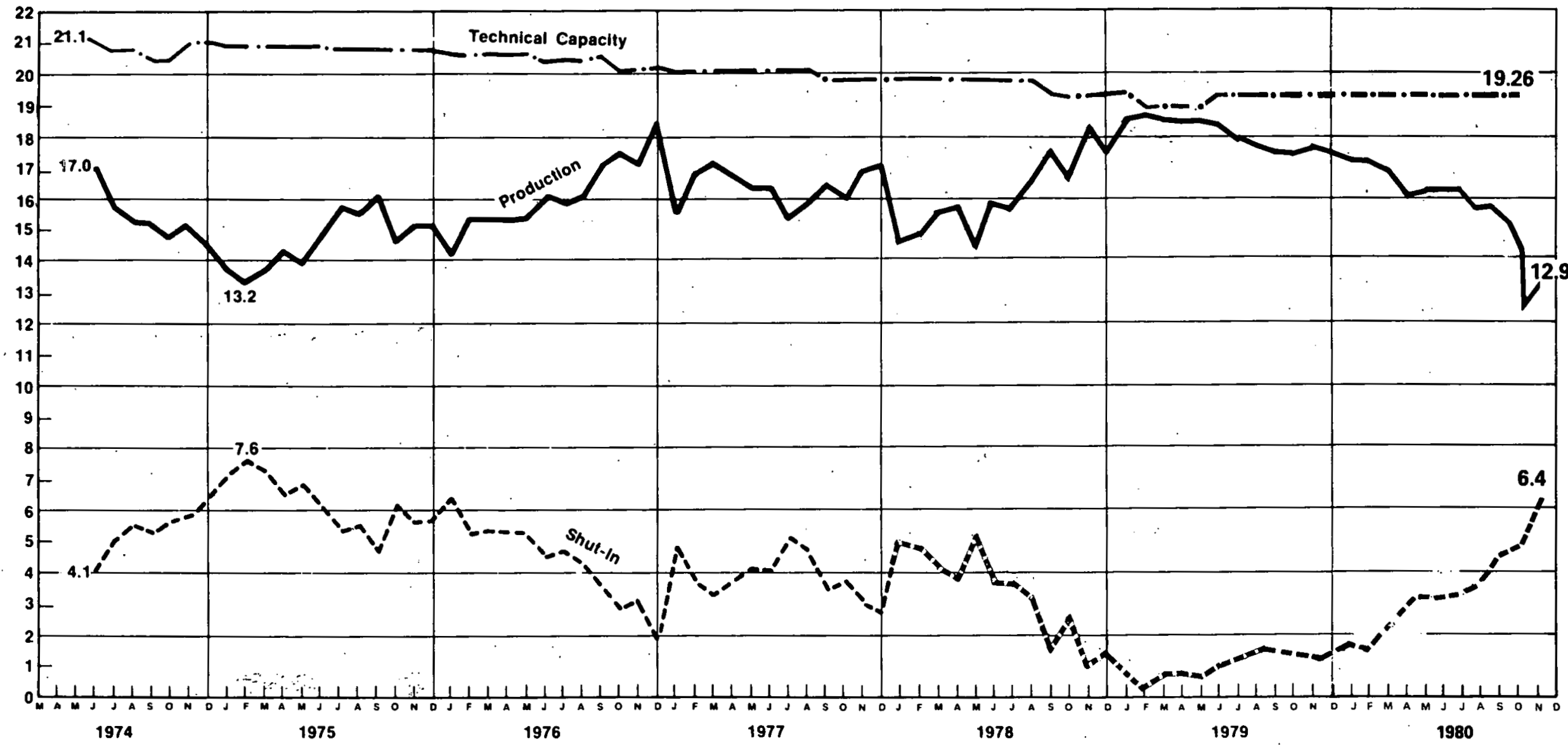


Oil Stocks: Free World, U.S., Japan and Europe (Landed), 1973 (In Million Barrels, End of Quarter)

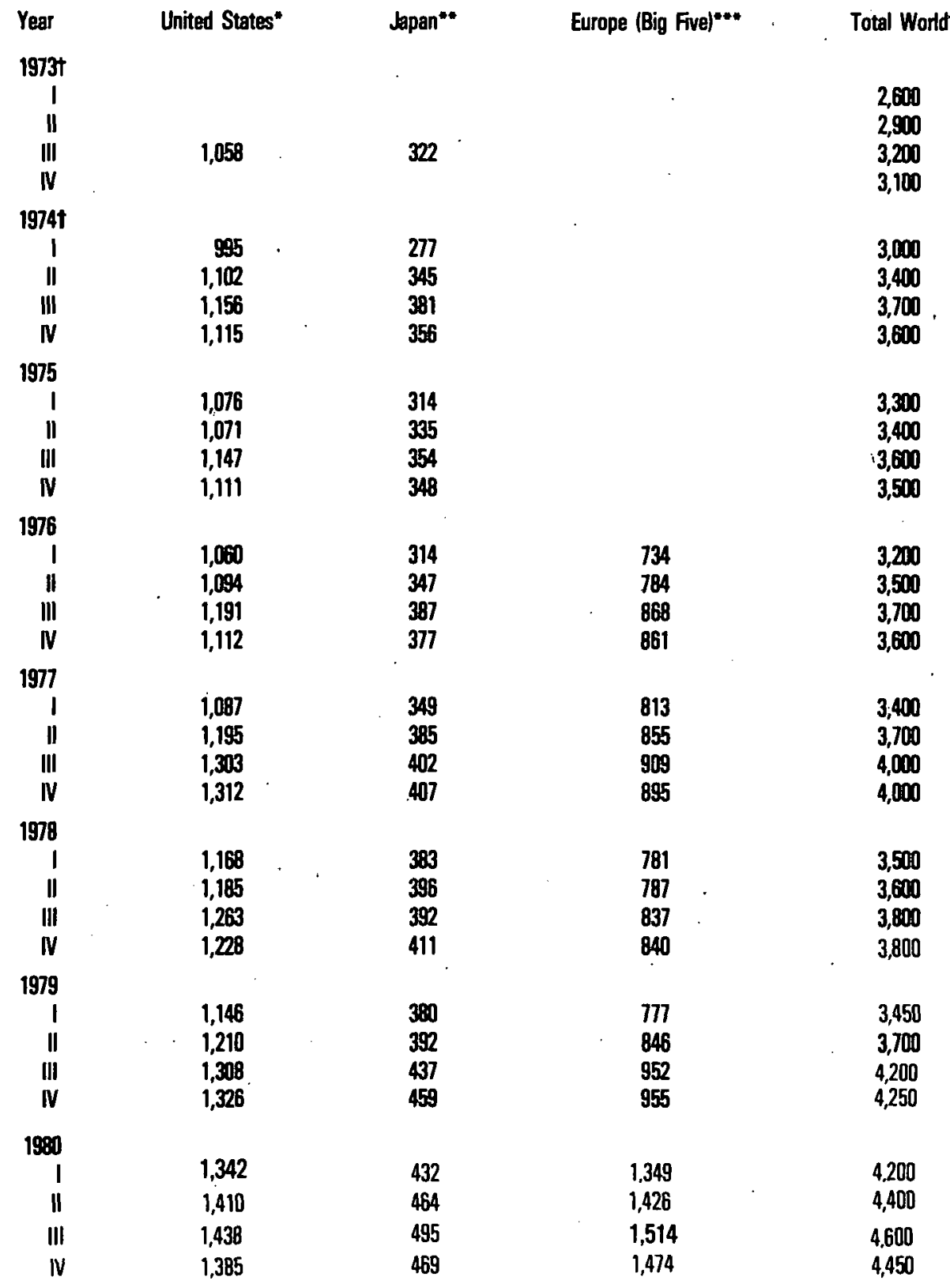

"U.S. stocks indude Strategic Petroteum Reserves which held 107.8 million bbl as of December 31, 1980.

(n)

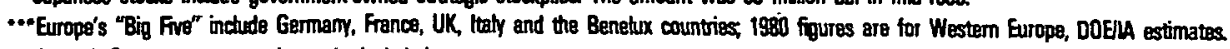

TEstimated. Government strategic stocks induded.

SOIrex: CIA. Energy Brandt, OECO; DOELA; Total Worth. DOEIA estimatas 
Oil Stocks: Free World, U.S., Japan and Europe, 1973 -

End of Quarter

Billion

bbl

(In Billions of Barrels)

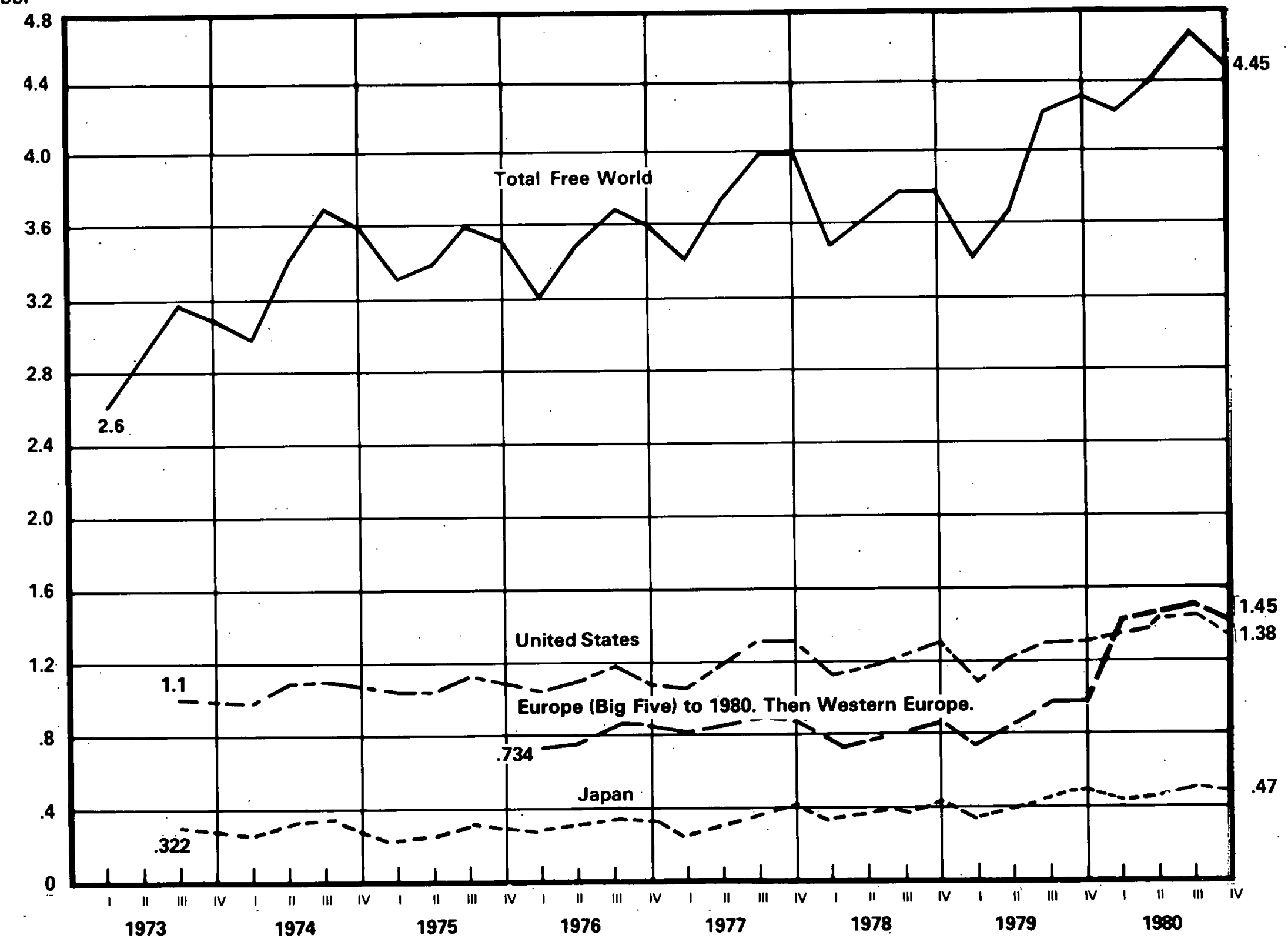




\section{Petroleum Consumption by Industrial Countries, 1973 - (In Thousands of Barrels a Day)}

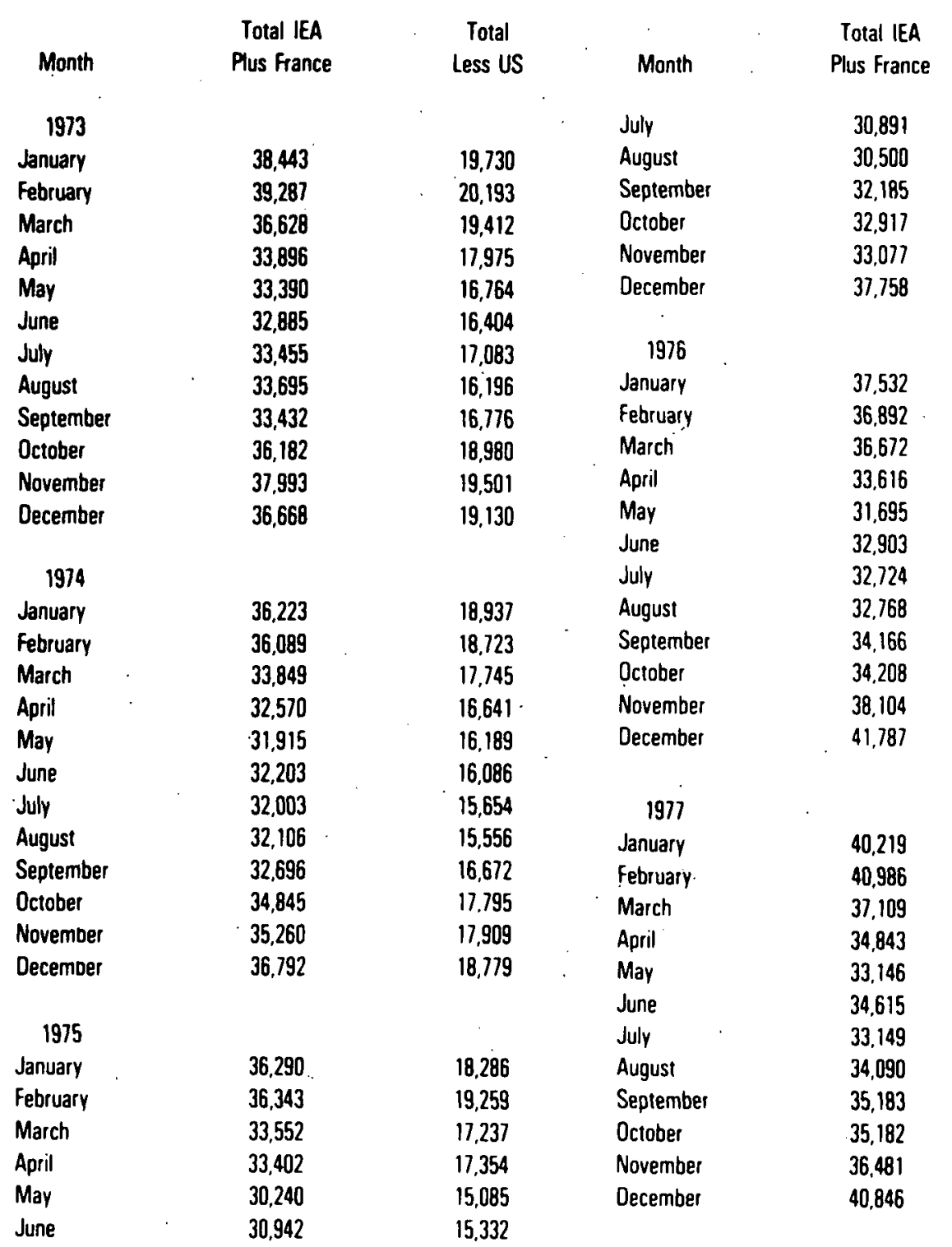

\begin{tabular}{|c|c|}
\hline $\begin{array}{l}\text { Total } \\
\text { Less US }\end{array}$ & Month \\
\hline 15,151 & 1978 \\
\hline 14,694 & January \\
\hline 16,417 & February \\
\hline 16,540 & March \\
\hline 17,300 & April \\
\hline 19,573 & May \\
\hline & June \\
\hline & July \\
\hline 18,934 & August \\
\hline 19,463 & September \\
\hline 19,373 & October \\
\hline 16,945 & November \\
\hline 15.718 & December \\
\hline 16,067 & \\
\hline 16,111 & 1979 \\
\hline 16.126 & $\mid y / y$ \\
\hline 17,341 & . January \\
\hline 17.156 & February \\
\hline 19,257 & March \\
\hline 21,281 & April \\
\hline & May \\
\hline & June \\
\hline & Juty \\
\hline 19,738 & Aungust \\
\hline 20,559 & September \\
\hline 19,053 & October \\
\hline 17.273 & November \\
\hline 16,186 & December \\
\hline 16,567 & \\
\hline 15,600 & 1980 \\
\hline 16,081 & January \\
\hline 17,450 & January \\
\hline 17,351 & Teoruary \\
\hline 18,041 & April \\
\hline 20,800 & May \\
\hline & June \\
\hline & July \\
\hline & August \\
\hline
\end{tabular}

Total IEA

Plus France

Total Less US

\section{9,745}

43,059

39,636

36,044

35,491

35,487

34,164

35,525

35,965

37,197

40,072

42,000

2719

19,984

18,297

17,201

17,227

16,531

16,886

18,011

18,780

20,916

22,056

42,686

22,029

22,586

$\begin{array}{ll}39,615 & 20,435 \\ 36,050 & 18,731\end{array}$

$36,139 \quad 18,425$

$35,263 \quad 17,598$

$34,604 \quad 17,549$

$36,253-18,069$

$35,521 \quad 18,251$

$37,407 \quad 19,283$

$38,781-20,519$

$37,278 \quad 18,495$

39,665

20,156

20,123

18,704

18,185

15,781

15,234

15,788

14,314

Source: CIA, Energy Branch; DOE Monthly Energy Review

-Consumption is that of the first 19 IEA countries plus France. Australia and Portugal joined the IEA later but not yet induded in the statistics.

Except for the U.S., consumption excludes bunkers, refinery fuel and losses. 


\section{Petroleum Consumption by Industrial Countries, 1973 -}

(in Millions of Barrels a Day)

\section{MMB/D}

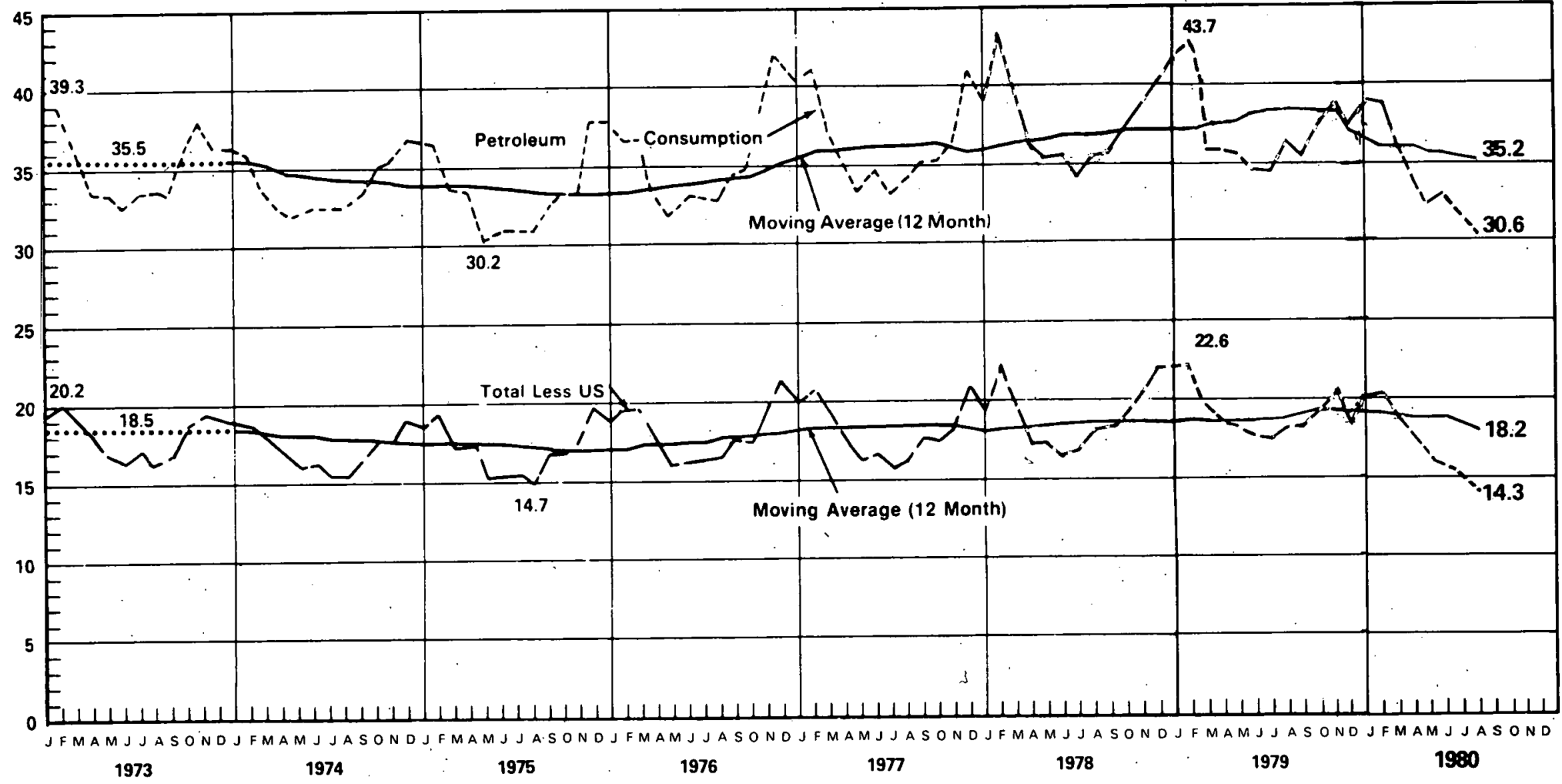


USSR Oil Production and Exports, 1975 (In Thousands of Barrels a Day)

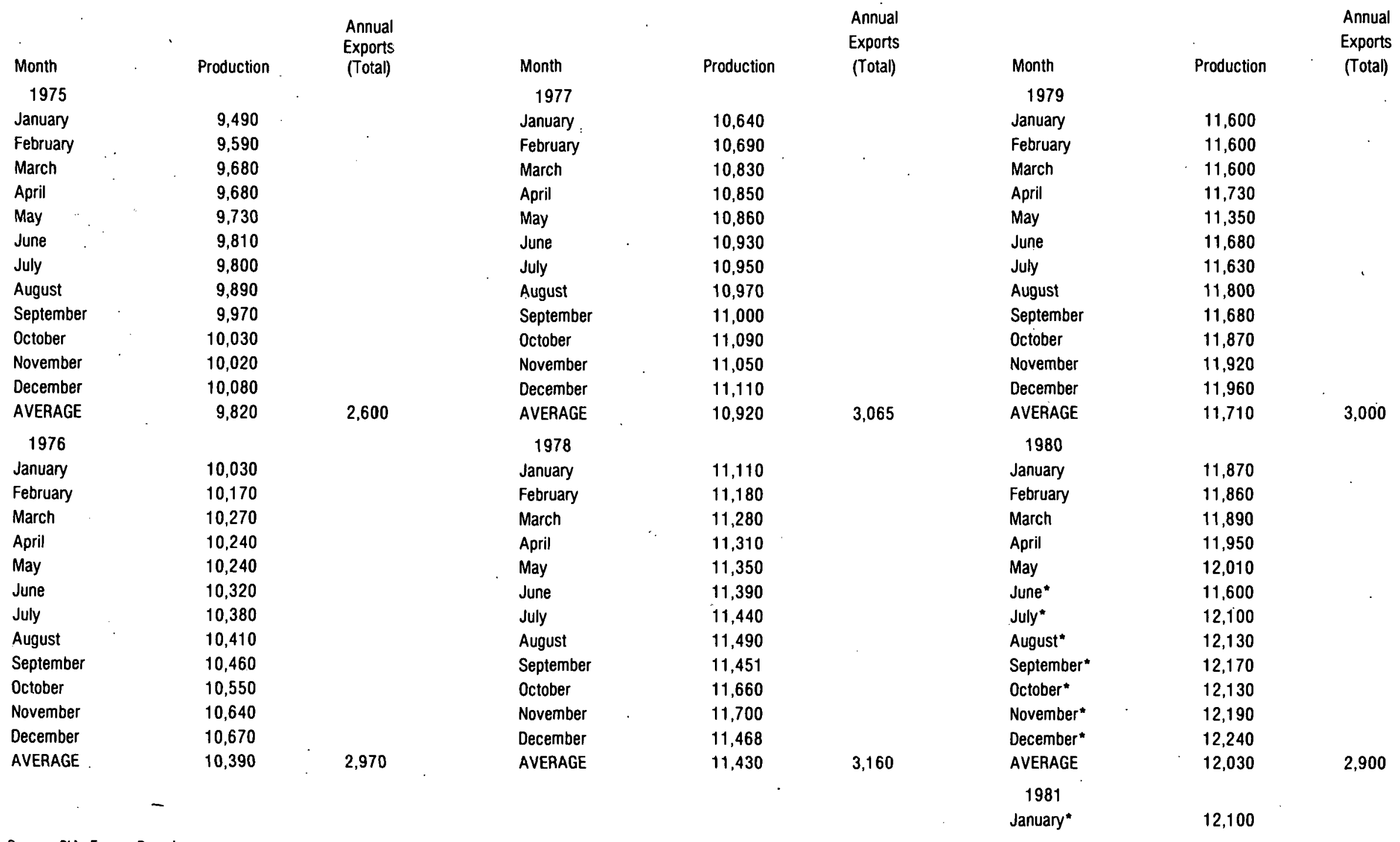

Source: CIA, Energy Branch

Note: Figures include natural gas liquids Export figures revised. Include and hence are not comparable with figures shown in Monthly Energy Review.

Bloc and Free World destinations.

Annual Totals as calculated by

- Preliminary estimates

CIA, adjusted 
USSR Oil Production, 1975 -

(Including Natural Gas Liquids)

Rate of Change in Production Over

Same Period of Preceding Year

\section{Percent}

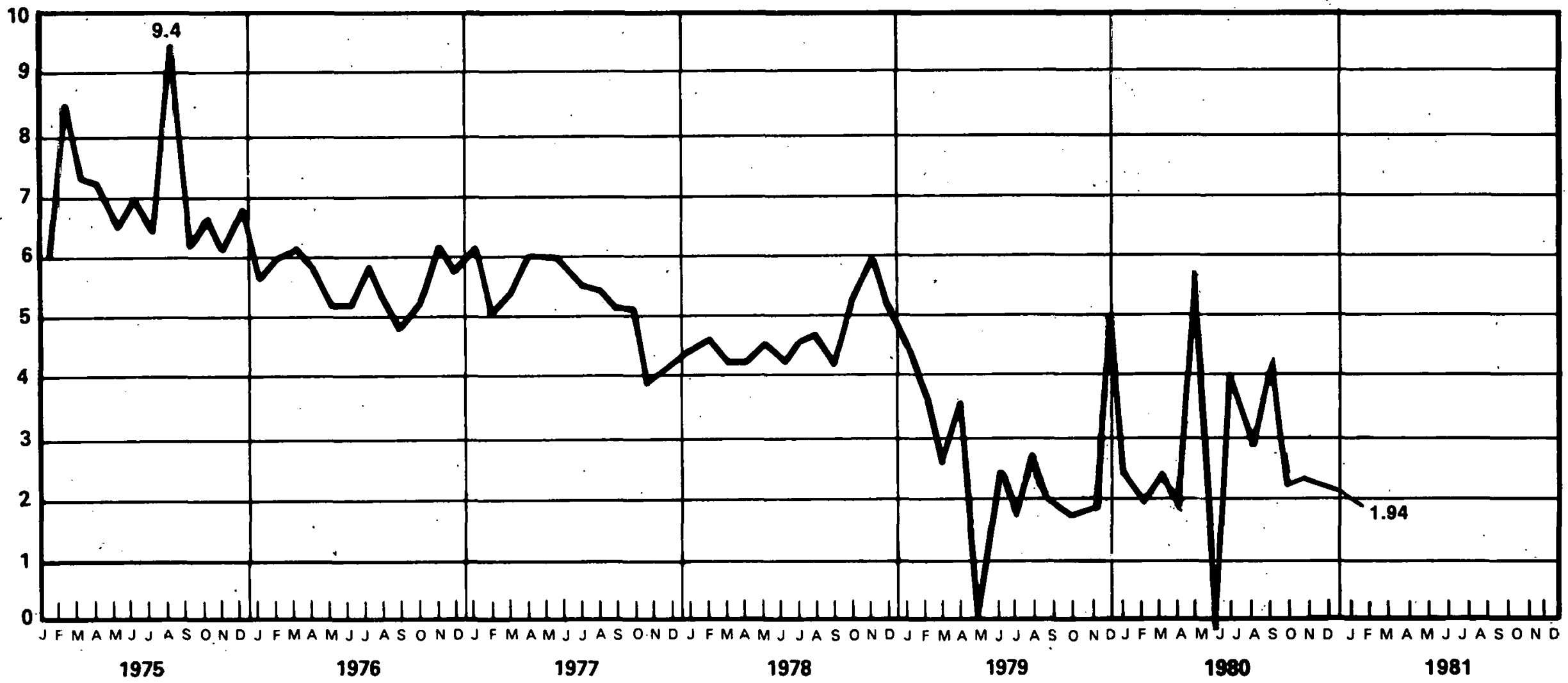


Free World and U.S. Nuclear Generation Capacity, 1973 (In Grosss Megawatts)

\begin{tabular}{|c|c|c|c|c|c|c|c|}
\hline Month & $\begin{array}{c}\text { Free World } \\
\text { Ex.US }\end{array}$ & $\begin{array}{l}\text { United } \\
\text { States }\end{array}$ & $\begin{array}{c}\text { Total } \\
\text { Free World }\end{array}$ & Month & $\begin{array}{c}\text { Free World } \\
\text { Ex-US }\end{array}$ & $\begin{array}{l}\text { United } \\
\text { States }\end{array}$ & $\begin{array}{c}\text { Total } \\
\text { Free World }\end{array}$ \\
\hline 1973 & & & & July & 27,064 & 37,334 & 64,398 \\
\hline January & - 18,109 & 15,449 & 33,558 & August & 27.984 & 37,330 & 65,314 \\
\hline February & 18,759 & 15,471 & 34,230 & September & 28,394 & 37,330 & 65,724 \\
\hline March & 18,779 & 15,471 & 34,250 & October & 28,953 & 38,130 & 67,083 \\
\hline April & 18,779 & 16,295 & 35,074 & November & 29,779 & 36,570 & 66,349 \\
\hline May & 19,237 & 16,295 & 35,532 & December & 29,792 & 39,399 & 69,191 \\
\hline June & 19,237 & 17,023 & 36,260 & & & & \\
\hline July & 19,237 . & $.17,023$ & 36,260 & 1976 & . & & \\
\hline August & 19,237 & 17,023 & 36,260 & January & 29,792 & 39,400 & 69,192 \\
\hline September & 19,237 & 17,910 & 37,147 & February & 29,770 & 39,350 & 69,120 \\
\hline October & 19,237 & 19,417 & 38,654 & March & 31,094 & 39,350 & 70,444 \\
\hline November & 19,237 & 19,417 & 38,654 & April & 31,094 & 39,370 & 70,464 \\
\hline \multirow[t]{2}{*}{ December. } & 19,714 & 20,887 & 40,601 & May & 32,394 & 41,240 & 73,634 \\
\hline & & & & June & 33,054 & 42,085 & 75,139 \\
\hline 1974 & & & & July & 33,057 & 42,715 & 75,782 \\
\hline January & 19,972 & 22,810 & 42,782 & August & 33,067 & 45,680 & 78,747 \\
\hline Febrùary & 19,976 & 23,648 & 43,624 & September & 34,729 & 43,200 & 77,929 \\
\hline March & 20,436 & 24,748 & 45,184 & October & 35,479 & 43,200 & 78,679 \\
\hline April & 20,436 & 25,208 & 45,644 & November. & 36,965 & 43,200 & 80,165 \\
\hline May & 20,428 & 25,991 & 46,419 & December & 36,965 & 44,080 & 81,045 \\
\hline June & 20,416 & 26,016 & 46,432 & . & & & \\
\hline July & 21,420 & 26,551 & 47,971 & 1977 & & & . \\
\hline August & 22,580 & 28,293 & 50,873 & January & 37,815 & 44,080 & 81,895 \\
\hline September & 22,580 & 29,180 & 51,760 & February & 37,815 & 44,910 & 82,725 \\
\hline October & 23,940 & 32,340 & 56,280 & March & 38,758 & 44,910 & 83,668 \\
\hline November & 24,667 & 32,827 & 57,494 & April & 40,304 & 46,090 & 86,394 \\
\hline \multirow[t]{2}{*}{ December } & 25.136 & 33,338 & 58,474 & May & 40,381 & 46,040 & 86,421 \\
\hline & & & & June & 40,381 & 46,230 & 86,611 \\
\hline 1975 & & & & July & 40,361 & 47,110 & 87,471 \\
\hline January & 26,454 & 34,626 & 61,080 & August & 40,301 & 46,930 & 87,231 \\
\hline February & 26,936 & 35,429 & 62,365 & September & 40,957 & 47,073 & 88,030 \\
\hline March & 26,569 & 35,446 & 62,015 & October & 41,858 & 47,311 & 89,169 \\
\hline April & 26,449 & 35,495 & 61,944 & November & 42,492 & 47,370 & 89,862 \\
\hline May & 27,064 & 36,495 & 63,559 & December & 43,352 & 48,340 & 91,692 \\
\hline June & 27,064 & 37,344 & 64,408 & & & & \\
\hline
\end{tabular}

Source: Nudeonics Week

\begin{tabular}{|c|c|c|c|}
\hline Monith & $\begin{array}{l}\text { Free World } \\
\text { Ex-US }\end{array}$ & $\begin{array}{l}\text { United } \\
\text { States }\end{array}$ & $\begin{array}{c}\text { Total } \\
\text { Free World }\end{array}$ \\
\hline \multicolumn{4}{|l|}{1978} \\
\hline January & 43,353 & 49,250 & 92,603 \\
\hline February. & 43,330 & 49,300 & 92,630 \\
\hline March & 43,350 & 50,430 & 93,780 \\
\hline April & 44,140 & 50,430 & 94,570 \\
\hline May & 45,100 & 50,470 & 95,570 \\
\hline June & 45,100 & 51,400 & 96,500 \\
\hline July & 45,130 & 51,400 & 96,530 \\
\hline August & 46,570 & 51,400 & 97,970 \\
\hline September & 46,570 & 52,360 & 98,930 \\
\hline October & 48,337 & 53,183 & 101,520 \\
\hline November & 51,030 & 53,140 & - 104,170 \\
\hline December & 50,940 & 54,120 & 105,060 \\
\hline \multicolumn{4}{|l|}{1979} \\
\hline January & 52,360 & 54,180 & 106.540 \\
\hline February & 53,300 & 54,180 & 107,460 \\
\hline March & 55,150 & 54,180 & 109,330 \\
\hline April & 56,230 & 54,180 & 110,410 \\
\hline May & 56,200 & 54,180 & 110,380 \\
\hline June & 56,200 & 54,180 & 110,380 \\
\hline Juty. & 56,190 & 54,220 & 110,410 \\
\hline August & 57,160 & 54,200 & 111,360 \\
\hline September & 58,470 & 54,200 & 112,670 \\
\hline October & 59,559 & 54,181 & 113,741 \\
\hline November & 61,703 & 54,193 & 115,896 \\
\hline December & 61,703 & 54,191 & 115,894 \\
\hline \multicolumn{4}{|l|}{1980} \\
\hline January & 61,452 & 54,191 & 115,643 \\
\hline February & 61,409 & 54,190 & 15,599 \\
\hline March & 63,323 & 54,192 & 117,515 \\
\hline Apri] & 64,223 & 54,185 & 118,400 \\
\hline May. & 64,224 & 54,191 & 118,415 \\
\hline June & 65,180 & 54,191 & 119,371 \\
\hline July & 65,180 & $.54,191$ & 119,371 \\
\hline August & 66,137 & 55,139 & 121,276 \\
\hline September & 67,117 & 55,139 & $.121,256$ \\
\hline October & 67,800 & 56,922 & 124,722 \\
\hline November & 68,485 & $.56,922$ & 125,407 \\
\hline December & 71,339 & 56,921 & 128,260 \\
\hline
\end{tabular}


Free World and U.S. Nuclear Generation Capacity, 1973 -

(In Thousand Gross Megawatts)

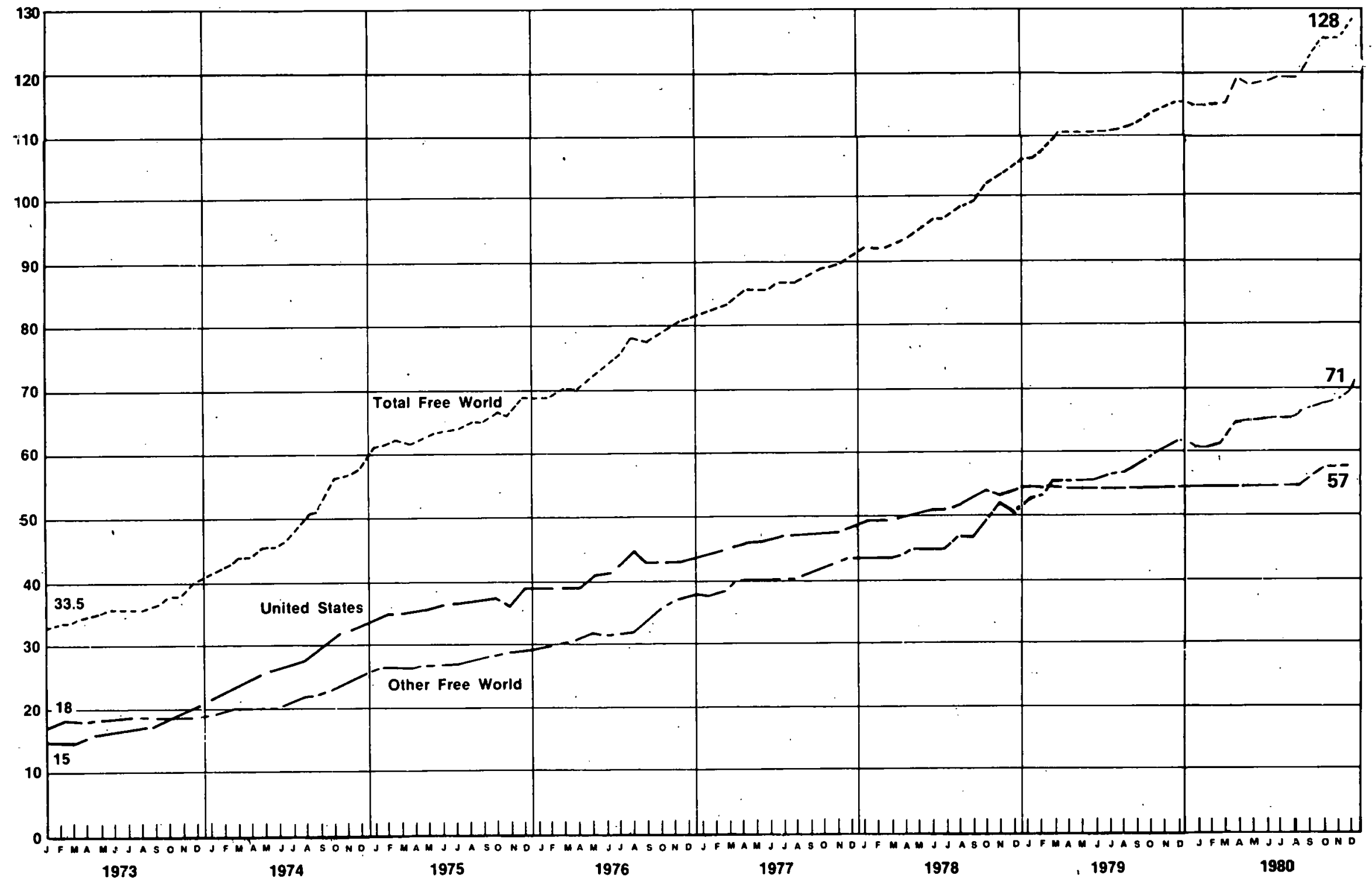


U.S. Gross Imports of Crude Oil and Products, 1973 (In Thousands of Barrels a Day)

\begin{tabular}{|c|c|c|c|c|c|c|c|c|c|c|c|}
\hline Month & $\begin{array}{l}\text { Crude } \\
\text { Oil }\end{array}$ & Products & Total & Month & $\begin{array}{c}\text { Crude } \\
\text { Oil }\end{array}$ & Products & Total & Month & $\begin{array}{l}\text { Crude } \\
\text { Oil }\end{array}$ & Products & Total \\
\hline 1973 AVERAGE & 3,244 & . 3,012 & 6,256 & $\begin{array}{c}1977 \\
\text { January }\end{array}$ & 6,281 & 2,622 & 8,903 & $\begin{array}{c}1979 \\
\text { January }\end{array}$ & 6,656 & 2,222 & 8,878 \\
\hline 1974 AVERAGE & 3,477 & 2,635 & 6,112 & February & 6,659 & 3,338 & 9,997 & February & 6,344 & 2,062 & $\begin{array}{l}0,070 \\
8,406\end{array}$ \\
\hline TOTP Notionol & & & & March & 6,699 & 2,684 & 9,383 & March & 6,240 & 2,385 & 8,625 \\
\hline 1975 & & & & April & 6,821 & 1,902 & 8,723 & April & 6,145 & 1,673 & 7,820 \\
\hline January & & & & May & 6,818 & 1,753 & 8,571 & May & 6,163 & 1,826 & 7,969 \\
\hline February & $\begin{array}{l}4,0<3 \\
3,828\end{array}$ & 2,832 & 6,861 & June & 7,065 & 1,872 & 8,937 & June & 6,554 & 1,672 & 8,226 \\
\hline March & $\begin{array}{l}3,0<0 \\
3,656\end{array}$ & 2,348 & 6.176 & July & 7,068 & 2,027 & 9,095 & July & 6,349 & 1,932 & 8,280 \\
\hline April & 3,378 & 2,074 & 5,730 & August & 6,395 & 2,179 & 8,574 & August & 6,774 & 1,778 & 8,552 \\
\hline May & 3,486 & 1,662 & 5,040 & September & 6,429 & 2,137 & 8,567 & September & 6,410 & 1,596 & 8,006 \\
\hline June & 3,905 & 1,728 & 5,214 & October & 6,409 & 1,862 & 8,272 & October & 6,854 & 1,785 & 8,639 \\
\hline July & 4,192 & 1,502 & 5,406 & November & 6.248 & 1,814 & 8062 & November & 6,154 & 1,946 & 8,099 \\
\hline August & & 1,767 & 5,959 & December & 6,248 & 2,198 & $\begin{array}{l}0,002 \\
8,446\end{array}$ & December & 6,273 & 2,305 & 8,577 \\
\hline September & 4,689 & 1,717 & 6,298 & AVERAGE & 6,594 & 2,193 & & AVERAGE & 6,411 & 1,933 & 8,344 \\
\hline October & 4,389 & $\begin{array}{l}2,115 \\
1,940\end{array}$ & 6,804 & & & & 8,787 & 1980 & & & \\
\hline November & 4,623 & $\begin{array}{l}1,940 \\
1,796\end{array}$ & $\begin{array}{r}6,329 \\
6,419\end{array}$ & 1978 & . & & & January & 6,359 & 1,983 & 8,342 \\
\hline December & 4,476 & 1,949 & $\begin{array}{l}6,419 \\
6,425\end{array}$ & January & 6,126 & 2,092 & 8,218 & February & 5,936 & 1,911 & 0,847 \\
\hline AVERAGE & 4,105 & 1,951 & 6,056 & February & 5,655 & 2,355 & 8,010 & March & 5,785 & 1,724 & 7,500 \\
\hline 1976 & & & & March & 6,031 & 2,338 & 8,369 & April & 5,555 & 1,430 & 6,985 \\
\hline January & 4.595 & & & April & 5,519 & 2,115 & 7,634 & May & 5,071 & . 1,478 & 6,549 \\
\hline February & 4,595 & 2,071 & 6,666 & May & 5,594 & 1,804 & 7,398 & June & 5,480 & 1,413 & 6,893 \\
\hline March & 4,208 & 2,423 & 6,631 & June & 6,322 & 1,640 & 7,962 & $\begin{array}{l}\text { July" } \\
\text { August" }\end{array}$ & 4,645 & 1,322 & 5,967 \\
\hline $\begin{array}{l}\text { Marcn } \\
\text { April }\end{array}$ & 4,738 & 1,945 & 6,683 & July & 6,175 & 1,948 & 8,123 & August" & 4,673 & 1,402 & 6,075 \\
\hline $\begin{array}{l}\text { April } \\
\text { May }\end{array}$ & 4,790 & 1,805 & 6,595 & August & 6,251 & 1,858 & 8,109 & September" & 4,569 & 1,420 & 5,989 \\
\hline $\begin{array}{l}\text { May } \\
\text { June }\end{array}$ & 4,669 & 1,654 & 6,323 & September & 6.829 & 1.983 & 8811 & October" & 4,500 & 1,289 & 5,789 \\
\hline $\begin{array}{l}\text { June } \\
\text { July . }\end{array}$ & 5,621 & 1,858 & 7,479 & October & 6,400 & 1,718 & & November" & 4,651 & 1,593 & 6,244 \\
\hline $\begin{array}{l}\text { July } \\
\text { August }\end{array}$ & 5,792 & 2,099 & 7,890 & November & 6,643 & $\begin{array}{l}1,1 / 18 \\
2,021\end{array}$ & $\begin{array}{l}8,119 \\
8,664\end{array}$ & December* & 5,038 & 1,804 & 6,842 \\
\hline $\begin{array}{l}\text { August } \\
\text { September }\end{array}$ & 5,556 & 1,826 & 7,382 & Decernber & 6,751 & $\begin{array}{l}2,021 \\
2,245\end{array}$ & $\begin{array}{l}8,664 \\
8,996\end{array}$ & 1981 & & & \\
\hline $\begin{array}{l}\text { September } \\
\text { October }\end{array}$ & 5,875 & 2,038 & 7,913 & AVERAGE & 6,195 & 2,008 & 8,996 & January* & $5135^{\circ}$ & 1003 & \\
\hline $\begin{array}{l}\text { Uctober } \\
\text { Novemiber }\end{array}$ & 5,699 & 1,808 & 7,508 & & & & 8,202 & & 0,135 & 1,903 & 7,038 \\
\hline December & $\begin{array}{l}5,946 \\
5,925\end{array}$ & 2,115 & 8,060 & & & & & · & & & \\
\hline AVERAGE & $\begin{array}{l}5,925 \\
5,287\end{array}$ & 2,468 & 8,393 & & & & & & & & \\
\hline & & & & & & & & & & & . \\
\hline
\end{tabular}


U.S. Gross Imports of Crude Oil and Products, 1975 -

(In Millions of Barrels a Day)

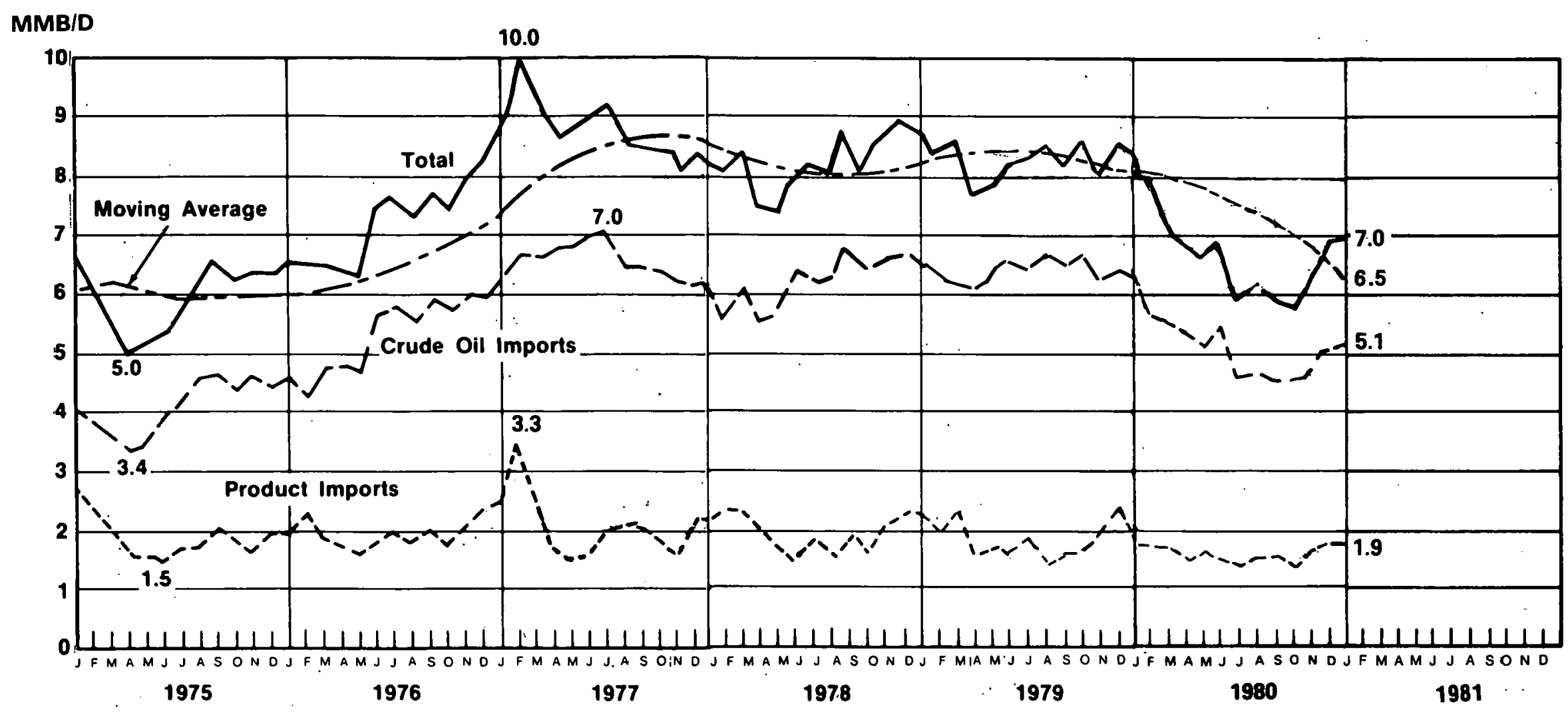

- Moving average is last 12 months.

- SPR purchases not included. 
Landed Cost of Saudi Crude Oil in Current and 1974 Dollars

(In Dollars per Barrel)

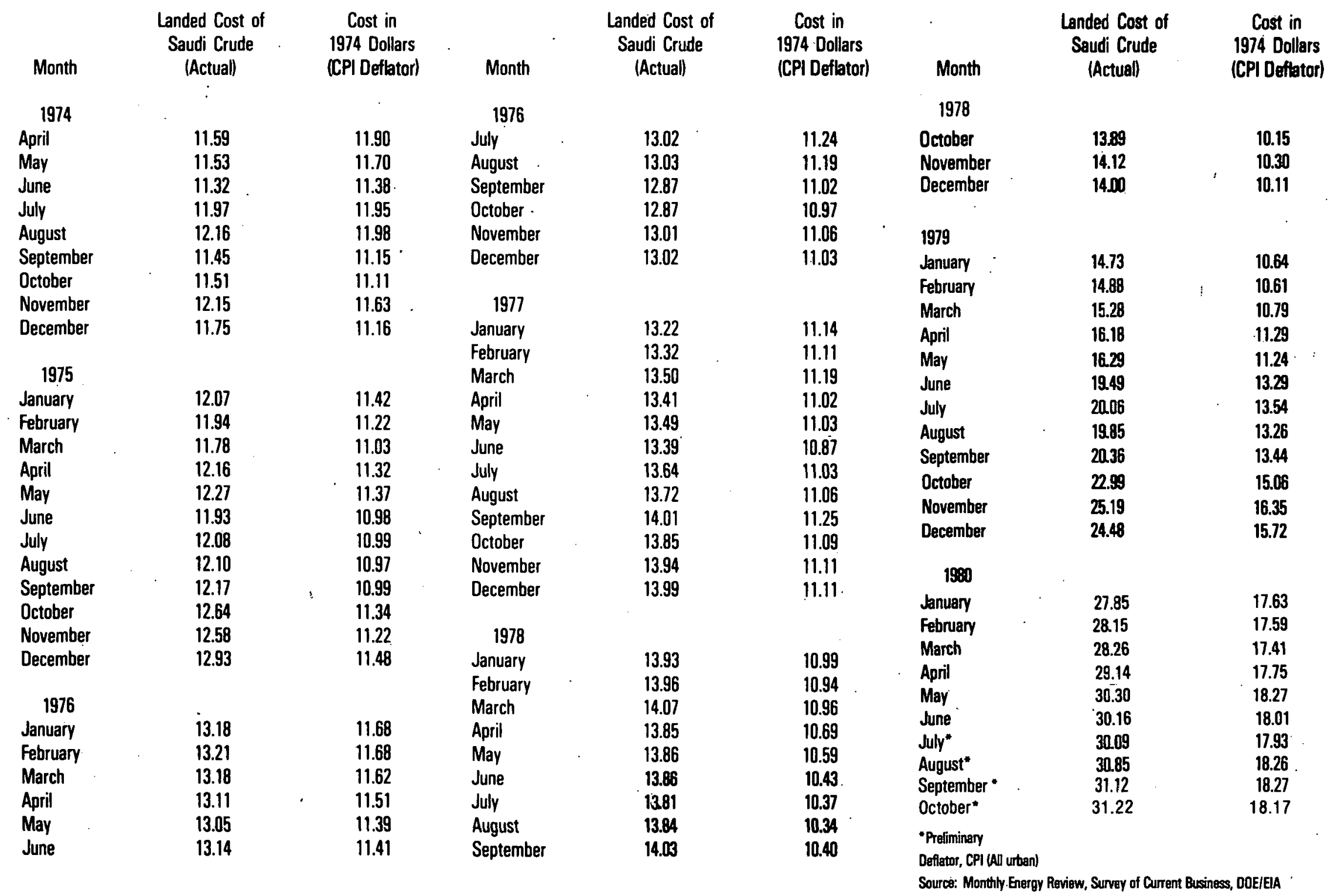




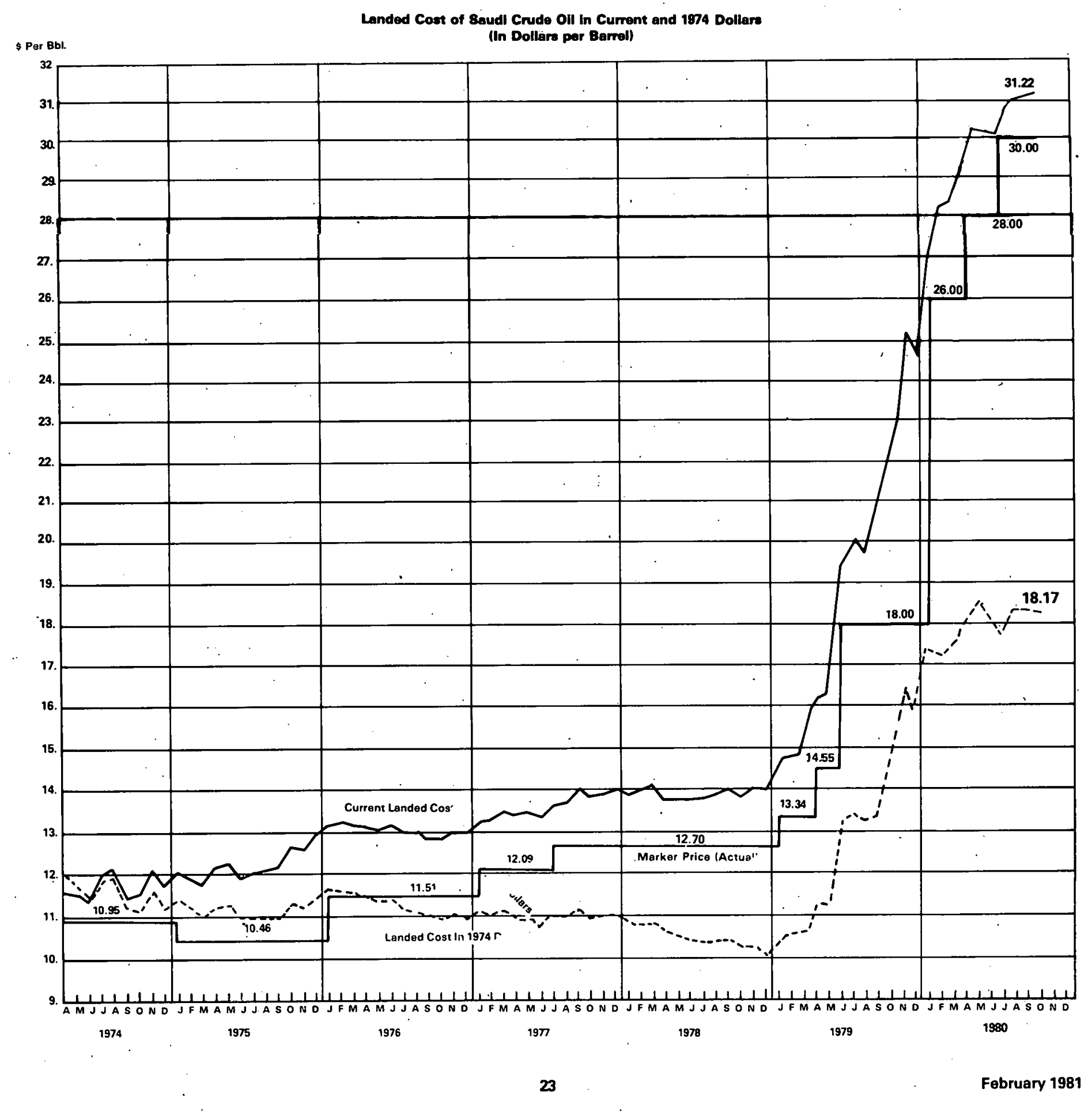


U.S. Trade in Bituminous Coal, 1973 (In Thousand Short Tons per Month)

\begin{tabular}{lcc} 
1973 & Exports & Imports \\
January & 2,954 & 34 \\
February & 2,669 & 31 \\
March & 3,377 & 36 \\
April & 5,063 & 40 \\
May & 5,140 & 125 \\
June & 4,969 & 102 \\
July & 4,188 & 76 \\
August & 5,133 & 96 \\
September & 3,424 & 187 \\
October & 5,882 & 218 \\
November & 5,214 & 153 \\
December & 4,889 & 115 \\
1974 & & \\
January & 2,813 & 333 \\
February & 4,627 & 344 \\
March & 3,179 & 346 \\
April & 4,944 & 424 \\
May & 6,032 & 437 \\
June & 6,369 & 387 \\
July & 5,307 & 402 \\
August & 5,088 & 780 \\
Septembel & 4,893 & 436 \\
October & 7,342 & 731 \\
November & 6,744 & 634 \\
December & 2,586 & 415 \\
1975 & & \\
January & 4,254 & 540 \\
February & 4,470 & 606 \\
March & 5,653 & 411 \\
April & 6,159 & 192 \\
May & 7,011 & 334 \\
June & 6,269 & 110 \\
July & 4,691 & 65 \\
August & 5,859 & 144 \\
September & 4,529 & 50 \\
October & 4,647 & 27 \\
November & 7,599 & 112 \\
December & 4,534 & .182 \\
\hline & & \\
\hline
\end{tabular}

\begin{tabular}{lcr} 
1976 & Exports & Imports \\
January & 3,697 & 118 \\
February & 3,050 & 139 \\
March & 3,979 & 54 \\
April & 5,780 & 156 \\
May & 5,667 & 116 \\
June & 6,569 & 265 \\
July & 4,880 & 134 \\
August & 4,223 & 361 \\
September & 5,613 & 261 \\
October & 5,871 & 413 \\
November & 5,451 & 291 \\
December & 4,625 & 225 \\
1977 & & \\
January & 2,143 & 149 \\
February & 3,079 & 130 \\
March & 3,390 & 67 \\
April & 5,637 & 200 \\
May & 5,673 & 203 \\
June & 6,019 & 257 \\
July & 5,159 & 304 \\
August & 4,279 & 274 \\
September & 5,037 & 458 \\
October & 4,871 & 595 \\
November & 4,491 & 503 \\
December & 3,910 & 514 \\
1978 & & \\
January & 870 & 139 \\
February & 555 & 159 \\
March. & 325 & 231 \\
April & 2,594 & 417 \\
May & 4,411 & 323 \\
June & 5,398 & 291 \\
July & 3,531 & 313 \\
August & 3,568 & 227 \\
September & 3,338 & 196 \\
October & 4,911 & 371 \\
November & 5,939 & 98 \\
December & 4,394 & 189 \\
& & \\
\hline
\end{tabular}

\begin{tabular}{lcc} 
1979 & Exports & Imports \\
January & $\mathbf{3 , 5 2 6}$ & 186 \\
February & 2,691 & 252 \\
March & $\mathbf{4 , 5 9 2}$ & 123 \\
April & $\mathbf{5 , 2 2 7}$ & 161 \\
May & $\mathbf{6 , 0 9 1}$ & 112 \\
June & $\mathbf{5 , 8 9 5}$ & 209 \\
July & $\mathbf{6 , 2 4 9}$ & 88 \\
August & $\mathbf{6 , 0 8 9}$ & $\mathbf{3 2 0}$ \\
September & $\mathbf{5 , 0 1 9}$ & 180 \\
October & $\mathbf{7 , 3 1 5}$ & 34 \\
November & $\mathbf{6 , 0 1 7}$ & 130 \\
Deccerniber & $\mathbf{8 , 0 7 2}$ & $\mathbf{1 4 8}$ \\
\hline
\end{tabular}

1980

January $\quad 4,292$

February $\quad 3,950 \quad 193$

March $\quad 5,565$

April $\quad 7,414 \quad 63$

May $\quad 8,449$

June $\quad 8,711 \quad 104$

July $\quad 7,972$

August $\quad 8,944 \quad 166$

Septemiber 8,265 2

$\begin{array}{lll}\text { October } & 9,204 & 97\end{array}$

$\begin{array}{llr}\text { November } & 8,905 & 3 \\ \text { December } & 8,169 & 70\end{array}$

Source: Burreau of Minas Oct. 7 forward, Bureau of the Census. 
U.S. Trade in Coal, 1973 -

(In Millions of Short Tons per Month)

Million

Short Tons

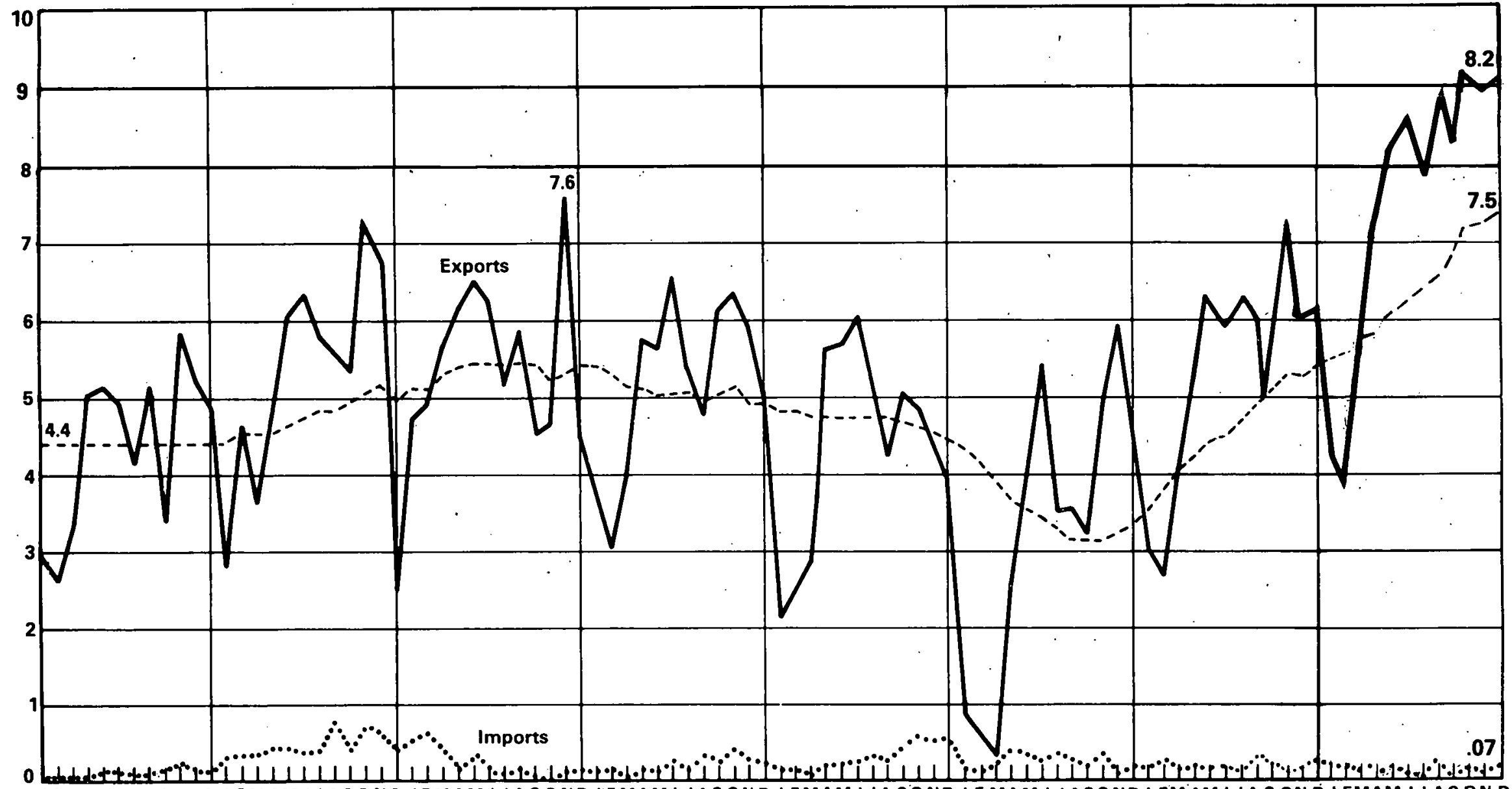

J FMAM J JAS OND J F MAMJ JASOND JFMAMJ JASONDJFMAMJ JASONDJFMAMJ JASOND J FMAMJ JASONDJ FMAMJ JAS ON DJ FMAMJJAS DN D

1973

1974

1975

1976

1977

1978

1979

1980 
Summary of U.S. Merchandise Trade, 1976 (In Millions of Dollars, Adjusted)

\begin{tabular}{|c|c|c|c|c|c|}
\hline \multicolumn{6}{|c|}{ EXPORTS } \\
\hline \multirow{2}{*}{\multicolumn{2}{|c|}{ Month }} & & & Manufactured & Agricuttural \\
\hline & & Total & Energy & Products & Products \\
\hline $\begin{array}{l}1976 \\
\text { Qutarter }\end{array}$ & 1 & 25,582 & 962 & 18.891 & 7397 \\
\hline & $\|$ & 28,195 & 1,113 & 19,995 & 7,087 \\
\hline & III & 28,787 & 1,029 & 20,277 & 7,481 \\
\hline & IV & 29,455 & 1,102 & 20,737 & 7,741 \\
\hline \multicolumn{2}{|l|}{ TOTAL" } & 113,318 & 4,226 & 79,990 & 29,102 \\
\hline \multicolumn{6}{|l|}{1977} \\
\hline \multirow[t]{4}{*}{ Quarter } & 1 & 28,853 & 880 & 20,425 & 7,548 \\
\hline & $\|$ & 29,902 & 1,148 & 20,812 & 7,942 \\
\hline & III & 29,915 & 1,143 & 21,487 & 7,285 \\
\hline & IV & 29,305 & 995 & 20,993 & 7.297 \\
\hline \multirow{2}{*}{\multicolumn{2}{|c|}{$\begin{array}{l}\text { TOTAL" } \\
1978\end{array}$}} & 117,963 & 4,179 & 83.678 & 30,106 \\
\hline & & & & & \\
\hline \multirow[t]{4}{*}{ Quarter } & 1 & 30,955 & 631 & 22,294 & 8,031 \\
\hline & 11 & 35,684 & 975 & 24,880 & 9,830 \\
\hline & III & 37,230 & 997 & 26,362 & 10,071 \\
\hline & IV & 39,635 & 1,178 & 28,502 & 9,955 \\
\hline \multicolumn{2}{|l|}{ TOTAL" } & 143,575 & 3,878 & 101,839 & 37,858 \\
\hline \multicolumn{6}{|l|}{1979} \\
\hline \multirow[t]{2}{*}{ Quarter } & 1 & 41,092 & 1,371 & 29,868 & 9,854 \\
\hline & II, & 42,783 & 1,309 & 30,675 & 10,797 \\
\hline \multicolumn{2}{|l|}{ July } & 15,669 & 538 & 10,876 & 4,255 \\
\hline \multicolumn{2}{|l|}{ August } & 15,821 & 499 & 11108 & 4,214 \\
\hline \multicolumn{2}{|c|}{ September } & 15,832 & 423 & 10,980 & 4,429 \\
\hline \multicolumn{2}{|l|}{ October } & 16,838 & 498 & 11,835 & 4,505 \\
\hline \multicolumn{2}{|l|}{ November } & 17,004 & 456 & 11,966 & 4,582 \\
\hline \multicolumn{2}{|l|}{$\begin{array}{l}\text { December } \\
1980\end{array}$} & 16,792 & 519 & 11,104 & 5,169 \\
\hline \multicolumn{2}{|l|}{ January } & 17,348 & 636 & 12,162 & 4,549 \\
\hline \multicolumn{2}{|l|}{ February } & 17,233 & 573 & 12,111 & 4,549 \\
\hline \multicolumn{2}{|l|}{ March } & 18,534 & 652 & 13,005 & 4,878 \\
\hline \multicolumn{2}{|l|}{ April } & 18,468 & 605 & 13,192 & 4,672 \\
\hline \multicolumn{2}{|l|}{ May } & 17,678 & 665 & 12,672 & 4,342 \\
\hline \multicolumn{2}{|l|}{ June } & 18,642 & 658 & 13,354 & 4,649 \\
\hline \multicolumn{2}{|l|}{ July } & 18,075 & 698 & 12,782 & 4,595 \\
\hline \multicolumn{2}{|l|}{ August } & 19,103 & 717 & 13,433 & 4,954 \\
\hline \multicolumn{2}{|c|}{ September } & 18,701 & 717 & 13,140 & 4,844 \\
\hline October & & 19,089 & 637 & 13,893 & 4,558 \\
\hline November & & 18,634 & 707 & 13,357 & 4,570 \\
\hline Decembe & & 19,118 & 689 & 13,400 & 5,029 \\
\hline
\end{tabular}

$\begin{array}{lrr}\begin{array}{l}\text { Month } \\ \text { I9ं76 }\end{array} & & \text { Total } \\ \text { Quarter } & \text { I } & \\ & \text { II } & 27,422 \\ & \text { III } & 28,874 \\ & \text { IV } & 31,834 \\ \text { TOTAL* } & 32,414 \\ \text { 1977 } & & 120,677 \\ \text { Quarter } & \text { I } & \\ & \text { II } & 35,149 \\ & \text { III } & 37,284 \\ & \text { IV } & 37,459 \\ \text { TOTAL* } & 37,264 \\ \text { 1978 } & & 146,817 \\ \text { Quarter } & \text { I } & \\ & \text { II } & 41,367 \\ & \text { III } & 42,471 \\ \text { IV } & 43,498 \\ \text { TOTAL* } & 183,137\end{array}$

IMPORTS

1979

Quarter 1

$\begin{array}{rcr}\text { Energy } & \begin{array}{c}\text { Manufactured } \\ \text { Products }\end{array} & \begin{array}{c}\text { Agricultural } \\ \text { Products }\end{array} \\ 7,448 & & \\ 7,749 & 15,530 & 4,441 \\ 9,305 & 16,486 & .4,639 \\ 9,490 & 17,504 & 5,025 \\ 33,996 & 17,755 & 5,169 \\ & 67,313 & 19,369 \\ 11,016 & & \\ 11,256 & 18,428 & 5,705 \\ 11,173 & 19,918 & 6,110 \\ 10,936 & 20,817 & .5,469 \\ 44,287 & 20,926 & 5,402 \\ & 79,895 & 22,635\end{array}$

\begin{tabular}{|c|c|c|c|c|}
\hline Quarter 1 & 46,310 & 11,170 & 27,655 & 6,955 \\
\hline$\|$ & 49,315 & 12,935 & 28,872 & 7,508 \\
\hline July & 16,777 & 5,075 & 9,425 & 2,277 \\
\hline August & 18,177 & 5,460 & 10,307 & 2,410 \\
\hline Septembèr & 18,666 & 6,084 & 10,204 & 2,377 \\
\hline October & 18,856 & 6,559 & 9,996 & 2,301 \\
\hline November & 18,422 & 5,411 & 10,370 & 2,641 \\
\hline $\begin{array}{l}\text { December } \\
1980\end{array}$ & 19,870 & 6,836 & 10,374 & 2,660 \\
\hline January & 22,107 & 6,895 & 12,313 & 2,900 \\
\hline February & 22,806 & 8,118 & 12,063 & 2,625 \\
\hline March & 21,692 & 7,744 & 11,194 & 2,754 \\
\hline April & 20,337 & 6,644 & 11,068 & 2,625 \\
\hline May & 21,637 & 7,176 & 11,851 & 2,610 \\
\hline June & 20,922 & 6,604 & 11.652 & 2,686 \\
\hline July & 19,926 & 6,011 & 11,265 & 2,650 \\
\hline August & 20,165 & 6,470 & 11,247 & 2,448 \\
\hline September & 20,345 & 6,053 & 11,881 & 2,410 \\
\hline October & 20,949 & 6,464 & 11,814 & 2,671 \\
\hline November & 20,297 & 6,091 & 11,416 & 2,790 \\
\hline December & 22,097 & 7,471 & 11,917 & 2,706 \\
\hline
\end{tabular}


Summary of U.S. Merchandise Trade, 1976 (In Billions of Dollars, Adjusted)
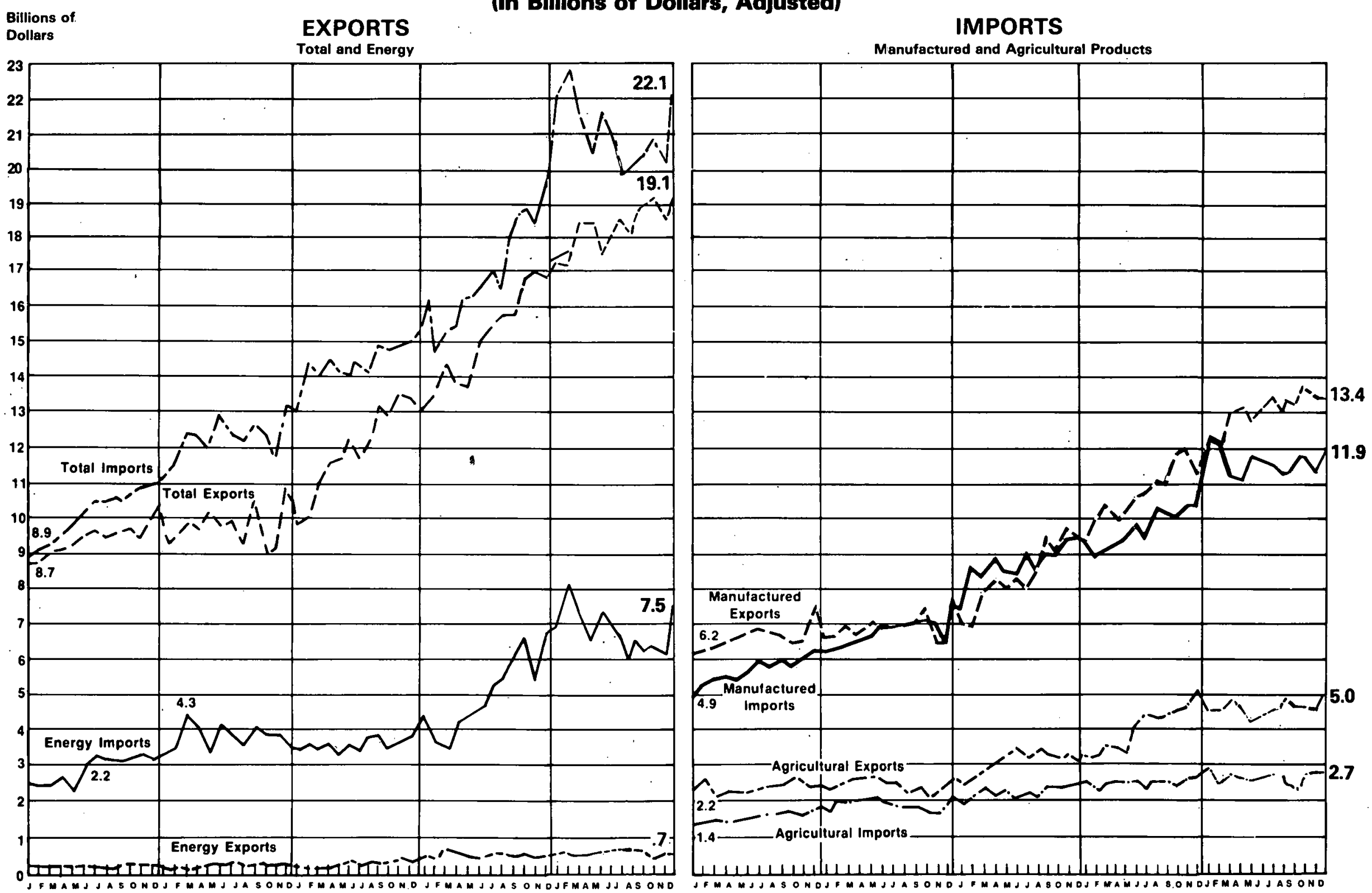


\section{U.S. Energy/GNP Ratio}

\section{(In 1972 Dollars)}

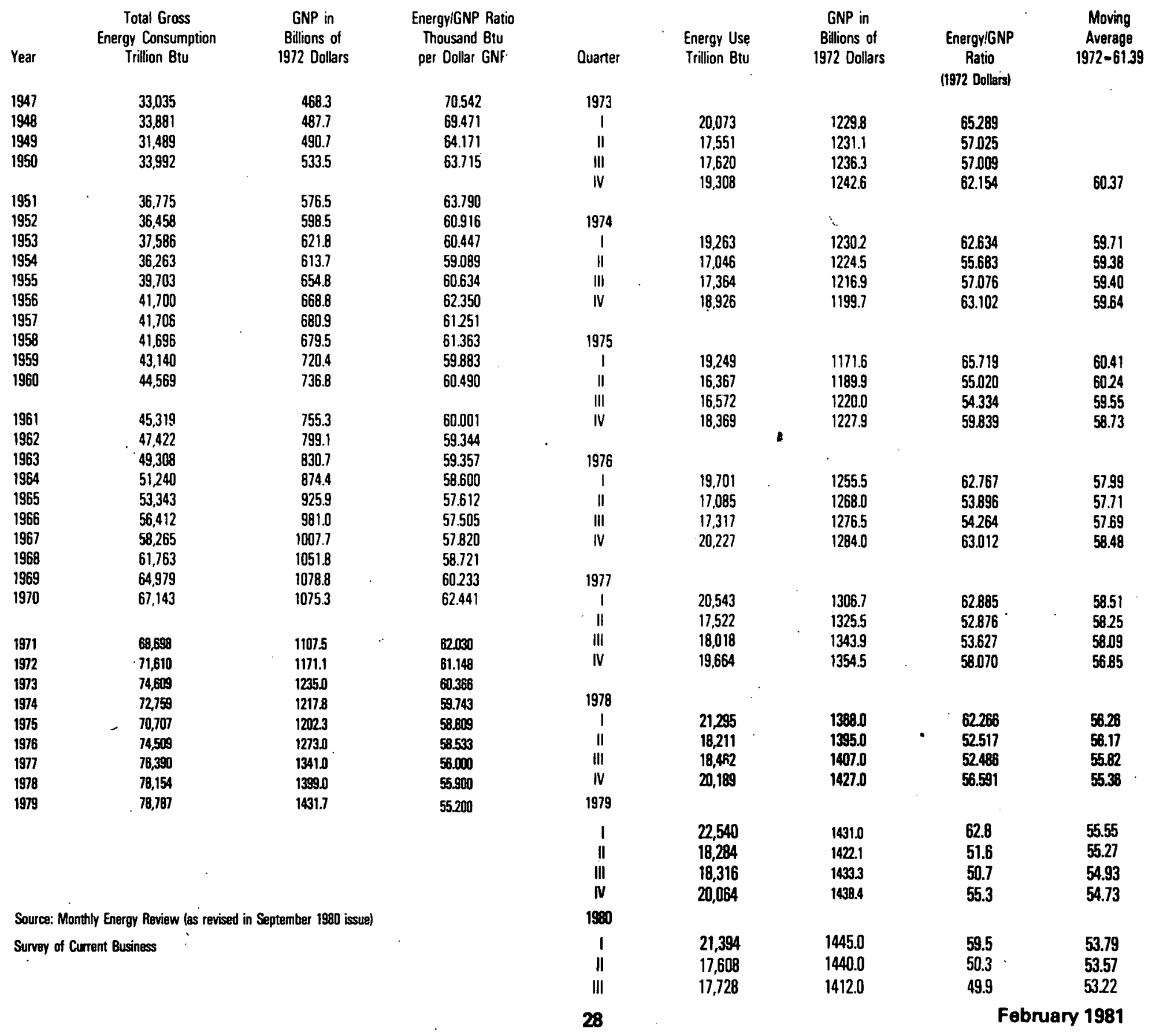




\section{U.S. Energy/GNP Ratio, 1972 Dollars}

1,000 Btu

per Dollar GNP

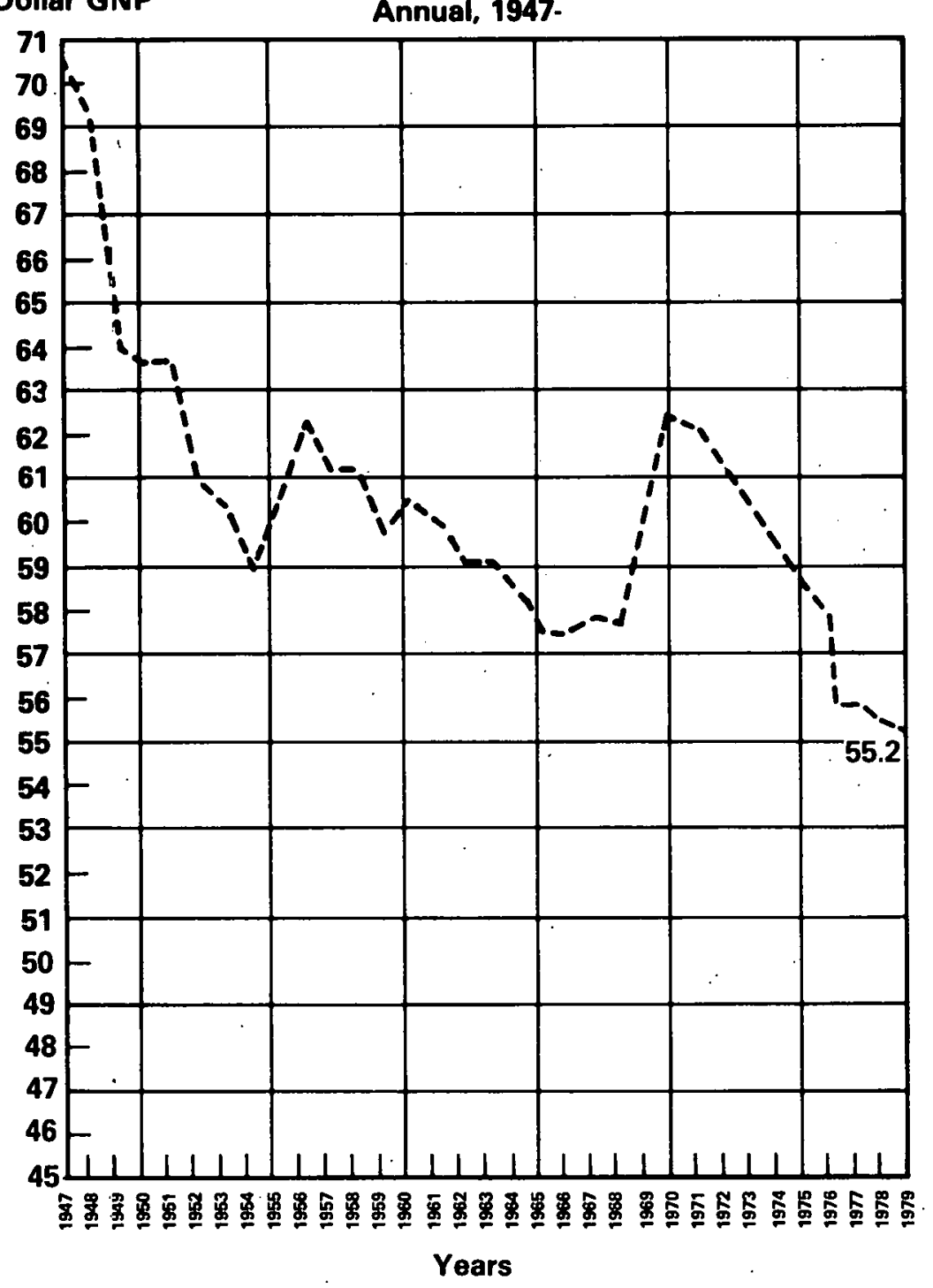

Quarterly, 1973 -

With Moving Average

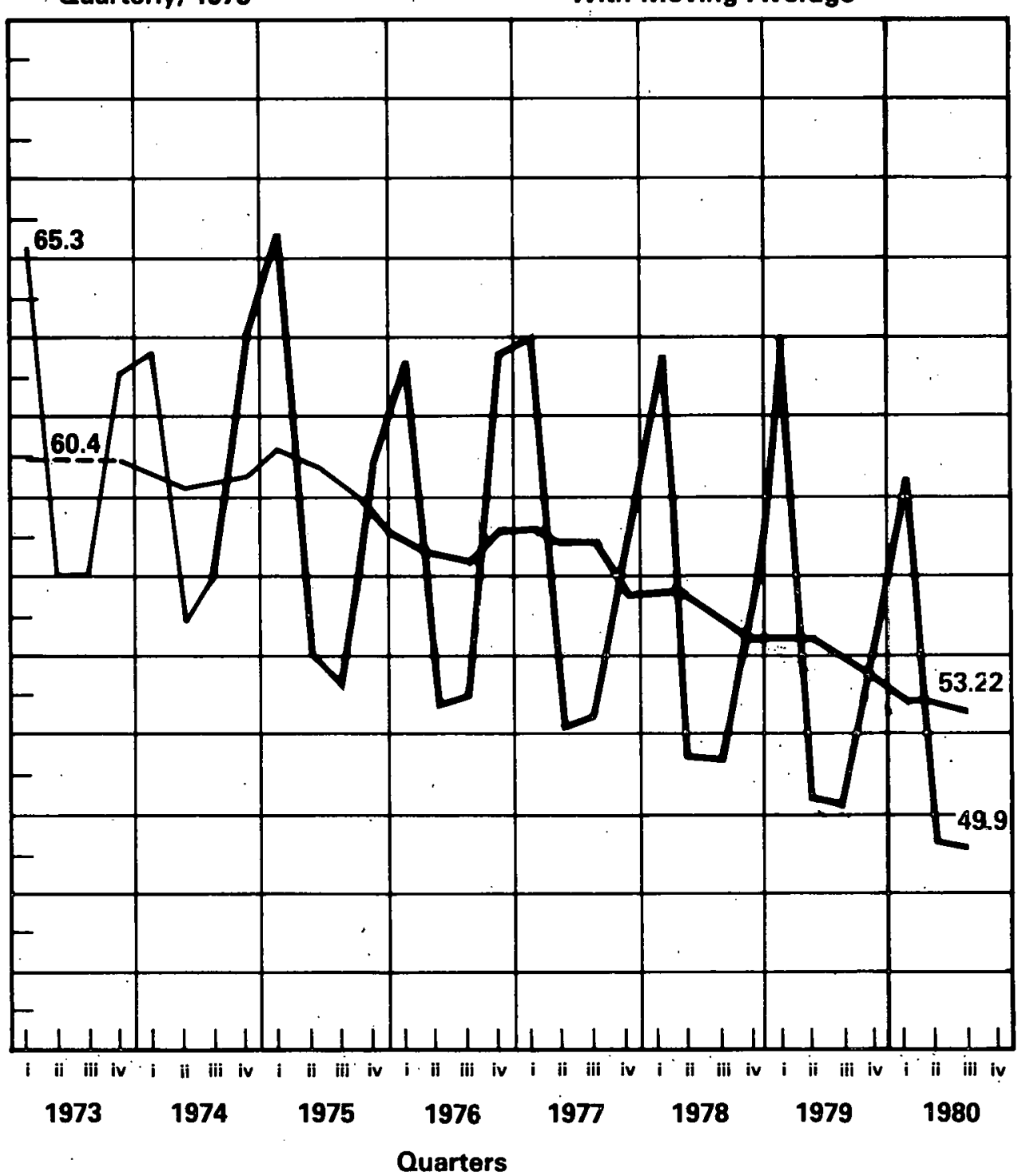

29 


\section{United States \\ Department of Energy \\ Washington, D.C. 20585}

Postage and Fees Paid

U.S. Departument of Energy

DOE-360

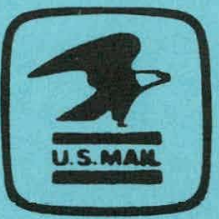

THIRD CLASS MAIL

Official Business

Penalty for Private Use, $\$ 300$ 